\title{
A general frailty model to accommodate individual heterogeneity in the acquisition of multiple infections: An application to bivariate current status data
}

\section{Thao M.P. Tran*1 | Steven Abrams ${ }^{1,2}$ | Roel Braekers ${ }^{1,3}$}

${ }^{1}$ Interuniversity Institute for Biostatistics and statistical Bioinformatics, Hasselt University, Diepenbeek 3590, Belgium

${ }^{2}$ Department of Epidemiology and Social Medicine, Wilrijk 2610, Belgium

${ }^{3}$ Interuniversity Institute for Biostatistics and statistical Bioinformatics, KU Leuven, Leuven 3000, Belgium

\section{Correspondence}

Thao M.P. Tran, Interuniversity Institute for Biostatistics and statistical Bioinformatics, Hasselt University, Diepenbeek 3590,

Belgium

Email: maiphuongthao.tran@uhasselt.be

\section{Funding information}

This work was supported by the Research Fund of Hasselt University (grant BOF11NI31 to S.A.). The authors also gratefully acknowledge financial support from the IAP research Network P7/06 of the Belgian Government (Belgian Science Policy) and the Methusalem research grant from the Flemish government.
The analysis of multivariate time-to-event (TTE) data can become complicated due to the presence of clustering, leading to dependence between multiple event times. For a long time, (conditional) frailty models and (marginal) copula models have been used to analyze clustered TTE data. In this paper, we propose a general frailty model employing a copula function between the frailty terms to construct flexible (bivariate) frailty distributions with the application to current status data. The model has the advantage to impose a less restrictive correlation structure among latent frailty variables as compared to traditional frailty models. Specifically, our model uses a copula function to join the marginal distributions of the frailty vector. In this paper, we considered different copula functions, and we relied on marginal gamma distributions due to their mathematical convenience. Based on a simulation study, our novel model outperformed the commonly used additive correlated gamma frailty model, especially in the case of a negative association between the frailties. At the end of the paper, the new methodology is illustrated on real-life data applications entailing bivariate serological survey data.

KEYWORDS 
Association; Copula functions; Frailty models; Maximum likelihood inference; Serological survey data.

\section{1 | INTRODUCTION}

Survival analysis techniques have become popular in many fields for their ability to deal with censoring and association in the analysis of time-to-event (TTE) data. Censoring occurs quite often in, for example, epidemiological and biomedical studies due to drop-out of study subjects or the absence of the event of interest before the end of the study (administrative censoring). Three types of censoring are often observed, i.e., left-censoring when the event occurred before the start of the study but the subject is included, right-censoring if the subject does not experience the event of interest before the time of censoring and interval-censoring whenever the event time is only known to have occurred somewhere in a certain interval. ${ }^{1}$ The latter situation takes place when, for example, subjects were observed at predefined monitoring times and the only available information is whether an event occurred between the previous visit and the current one or not. One particular type of interval-censored data, which is of primary interest for the applications in this paper, is Type I interval censored or so-called current status (CS) data, arising when subjects are examined only once. ${ }^{2}$ In case of CS data $(T, \Delta)$, the true event times are not know. The only knowledge about the true event time $T^{*}$ is that $T^{*} \leq T\left(T^{*}>T\right)$ when the censoring indicator $\Delta$ is equal to $1(0)$, where $T$ denotes the examination (or monitoring) time.

The analysis of univariate TTE data could be done using nonparametric, semi-parametric or parametric approaches which have been studied extensively in the survival literature (see for example, ${ }^{1,3}$ ). These techniques rely on the assumption of independent event times across and within study subjects. In practice, however, this independence assumption is questionable. For example, in studies collecting the time until tumor occurrence in twins, in experiments studying the occurrence of eye infections in both the right and left eye of a single person, and in serological studies describing the acquisition of multiple infections within the same person which are potentially transmitted via the same route. Consequently, in such cases, we need to use appropriate techniques to deal with dependency arising from clustering (e.g. within twins, or individuals). Due to the presence of clustering, the analysis of bivariate, and more general multivariate TTE data can become complicated. In the literature, the analysis of multivariate TTE data is done by either using a (conditional) frailty or (marginal) copula model. The copula model is attractive in the sense that one can model and estimate the association and the margins separately. ${ }^{4}$ Many authors have studied copula models to analyze bivariate survival data. Shil and Louis ${ }^{4}$ presented the performance of a few families of bivariate distributions (Clayton, Gumbel, and Frank) in copula models and compared the performance of the two-stage estimation approach to maximum likelihood estimation (MLE). Recently, Romeo et al. ${ }^{5}$ used the Bayesian framework to analyze bivariate survival analysis using the power variance function (PVF) copula, while considering a one-stage estimation approach. Other authors, for example, Andersen ${ }^{6}$ and Prenen et al. ${ }^{7}$ made use of two-stage frequentist approaches for estimating the aforementioned PVF copula and the marginal distributions. Lawless and Yilmaz ${ }^{8}$ used copula-based models to analyze recurrent failure time data. The method was then extended by Meyer and Romeo ${ }^{9}$ to model more than two recurrent events within a Bayesian framework. Our manuscript, however, focuses on the analysis of bivariate current status data in the absence of event recurrences, albeit that extensions of this approach could be of interest in those settings as well.

Although copula-based models are useful to study the population effects, they provide no insight into individual heterogeneity. Among the methods used to model the association between event times within the same subject, or event times corresponding to members of the same cluster, frailty models have become progressively popular. ${ }^{1}$ Frailty 
models provide an appealing tool to explicitly model the association between event times within the same cluster as well as the heterogeneity between clusters. Frailty models are conditional models in the sense that they condition on latent frailty terms, following a specific (frailty) distribution, which acts multiplicatively on a baseline hazard function. It is worth emphasizing that the copula and frailty models are quite different in nature. ${ }^{10}$ The frailty approach offers an elegant way to account for unobserved individual heterogeneity, as the frailty terms have a biological, medical and epidemiological meaning (reflecting, e.g., variation in social activity, and individual susceptibility to infection). This feature is vital to account for reliable estimates of important epidemiological parameters that are frequently used in infectious disease modeling to inform outbreak prevention and disease. ${ }^{11}$ Many frailty distributions have been proposed and extensively studied in the survival literature such as the lognormal distribution and members of the PVF distribution (e.g., gamma, inverse Gaussian, etc.), among others. ${ }^{12}$ Among the aforementioned distributions, the gamma distribution is the most popular one due to its closed-form expression for the Laplace transform, and hence, the marginal survival functions. ${ }^{13}$

Bivariate shared and correlated (gamma) frailty models have been employed to analyze paired current status data in infectious disease modeling (i.e., two infection statuses measured in the same individual) thereby describing the association between infection-specific frailty terms. ${ }^{12,14,15}$ In general, the main difference between these models lies in the specification of the bivariate frailty distribution and, equivalently, the implied correlation between the eventspecific frailties. A perfect positive correlation is assumed in a shared frailty model whereas the correlated gamma counterpart, allows for a non-trivial non-negative correlation between the infection-specific frailties. ${ }^{16}$ However, the implied correlation coefficient in the model by Yashin et al. ${ }^{16}$ exhibits a restrictive upper bound whenever the frailty variances are very different due to the additive decomposition of the frailties.

In this paper, we propose a general frailty model, unifying concepts from both copula and frailty approaches. More specifically, a bivariate copula function is employed to construct a flexible bivariate frailty distribution, thereby still implying a (conditional) frailty model. Our proposed model has a clear advantage over the correlated (gamma) frailty model in the sense that it is more flexible regarding the imposed correlation structure thereby containing the correlated model mentioned earlier as a special case. For the data applications in this manuscript, our general frailty model is applied to paired current status data in the field of infectious disease modeling. The performance of our proposed model is evaluated in comparison with correlated gamma frailty models. The paper is organized as follows. We first introduce our motivating examples in Section 2. In Section 3, the novel general frailty methodology is described in detail. Next, a simulation study is performed to compare the new model with existing frailty models when analyzing bivariate current status data (Section 4). In Section 5, we show the results of applying the new model to the data introduced in Section 2. We formulate conclusions and potential avenues for further research in Section 6.

\section{2 | MOTIVATING EXAMPLES}

A seroprevalence survey on a large representative national serum bank was conducted in 2002 in Belgium. The serological survey data were obtained from residual sera submitted for routine laboratory testing. More specifically, blood samples were tested for the presence of Immunoglobulin G (IgG) antibodies against varicella-zoster virus (VZV; also known as chickenpox), parvovirus B19 (PVB19; or fifth disease) and hepatitis A (HAV) infections, among other pathogens, using ELISA tests. IgG concentrations were dichotomized to indicate whether individuals were seronegative (not yet infected with the pathogen) or seropositive (an indication of past infection) based on prespecified infection-specific cut-off values. Hence, ignoring diagnostic uncertainty, the data constitutes CS data on past infection for the different pathogens under study. Based on these multi-sera data, the population-level seroprevalence 
can be determined, at least under the assumption of time equilibrium and lifelong humoral immunity upon contraction of the disease. ${ }^{18}$ In general, samples of individuals aged six months up to 71 years were present. Depending on the disease under consideration, 3256, 3080 and 3374 individuals had an observed immunological status for VZV, PVB19, and HAV, respectively. These three diseases have different transmission routes. While VZV and PVB19 are childhood infections that are mainly transmitted via infected respiratory droplets, HAV is transmitted primarily by the fecal-oral route. Throughout this paper, we analyzed two combinations of paired infections in individuals, namely data on (1) VZV and PVB19 infections, and (2) VZV and HAV infections. Note that we only use the complete cases (individuals with infection status for both diseases under consideration).

\section{3 | MATERIALS AND METHODS}

In this section, we first introduce the frailty terminology used throughout this paper. Next, we briefly discuss bivariate frailty models and bivariate copula functions. Finally, we describe our novel general frailty model thereby highlighting several special cases of the general model, and we show how these models can be applied in the context of bivariate CS data as introduced in Section 2.

\subsection{Frailty terminology for bivariate TTE data}

Let $\left(T_{1}^{*}, T_{2}^{*}\right)$ represent the vector of event times for two related events (for example, the time to a specific disease for the members of a twin pair or the time to two different infections in the same individual). In this paper, we consider two events (infections) within the same individual, thereby considering the individual as a cluster. A bivariate frailty model can be formulated in terms of the conditional hazard function for event (infection) $i=1,2$, say $\lambda_{i}\left(t_{i}^{*} \mid Z_{i}, \boldsymbol{\theta}_{\boldsymbol{i}}\right)$, at time $t_{i}^{*}$, conditional on frailty term $Z_{i}$, and depending on the set of parameters $\boldsymbol{\theta}_{\boldsymbol{i}}$. Under the commonly made proportional hazards assumption, the conditional hazard function decomposes into $\lambda_{i}\left(t_{i}^{*} \mid Z_{i}, \boldsymbol{\theta}_{\boldsymbol{i}}\right)=Z_{i} \lambda_{i 0}\left(t_{i}^{*} \mid \boldsymbol{\theta}_{\boldsymbol{i}}\right)$, where $\lambda_{i 0}\left(t_{i}^{*} \mid \boldsymbol{\theta}_{\boldsymbol{i}}\right)$ is the so-called baseline hazard function. Based on the conditional hazards, the conditional univariate survival functions can be written as ${ }^{11}$ :

$$
S_{i}\left(t_{i}^{*} \mid Z_{i}, \boldsymbol{\theta}_{\boldsymbol{i}}\right)=\exp \left(-Z_{i} \int_{0}^{t_{i}^{*}} \lambda_{i 0}\left(u \mid \boldsymbol{\theta}_{\boldsymbol{i}}\right) d u\right)=\exp \left[-Z_{i} \wedge_{i 0}\left(t_{i}^{*} \mid \boldsymbol{\theta}_{\boldsymbol{i}}\right)\right]
$$

where $\Lambda_{i 0}\left(t_{i}^{*} \mid \boldsymbol{\theta}_{\boldsymbol{i}}\right)=\int_{0}^{t_{i}^{*}} \lambda_{i 0}\left(u \mid \boldsymbol{\theta}_{\boldsymbol{i}}\right) d u$ denotes the cumulative or integrated baseline hazard function. Typically, one assumes independence of the event times $T_{1}^{*}$ and $T_{2}^{*}$ given the frailties $Z_{1}$ and $Z_{2}$ (conditional independence assumption) which implies that the joint survival function can be rendered as:

$$
S\left(t_{1}^{*}, t_{2}^{*} \mid Z_{1}, Z_{2}, \boldsymbol{\theta}\right)=S_{1}\left(t_{1}^{*} \mid Z_{1}, \boldsymbol{\theta}_{1}\right) S_{2}\left(t_{2}^{*} \mid Z_{2}, \boldsymbol{\theta}_{2}\right)=\exp \left[-Z_{1} \wedge_{10}\left(t_{1}^{*} \mid \boldsymbol{\theta}_{1}\right)\right] \exp \left[-Z_{2} \wedge_{20}\left(t_{2}^{*} \mid \boldsymbol{\theta}_{2}\right)\right]
$$

Integrating out the random frailty terms over the marginal and joint frailty distributions $f_{Z_{1}}, f_{Z_{2}}$ and $f$, respectively, yields expressions for the unconditional (or marginal) survival functions. One can write these expressions in terms of the univariate and bivariate Laplace transforms, denoted by $\mathcal{L}_{i}().(i=1,2)$ and $\mathcal{L}(.$, .), respectively, associated with the aforementioned frailty distributions, i.e., $S_{i}\left(t_{i}^{*} \mid \boldsymbol{\theta}_{\boldsymbol{i}}\right)=\mathrm{E}_{Z_{i}}\left[S_{i}\left(t_{i}^{*} \mid Z_{i}, \boldsymbol{\theta}_{\boldsymbol{i}}\right)\right]=\mathcal{L}_{i}\left[\Lambda_{i 0}\left(t_{i}^{*} \mid \boldsymbol{\theta}_{\boldsymbol{i}}\right)\right]$ and $\boldsymbol{S}\left(t_{1}^{*}, t_{2}^{*} \mid \boldsymbol{\theta}\right)=$ $\mathrm{E}_{Z_{1} Z_{2}}\left[S\left(t_{1}^{*}, t_{2}^{*} \mid Z_{1}, Z_{2}, \boldsymbol{\theta}\right)\right]=\mathcal{L}\left[\Lambda_{10}\left(t_{1}^{*} \mid \boldsymbol{\theta}_{1}\right), \Lambda_{20}\left(t_{2}^{*} \mid \boldsymbol{\theta}_{2}\right)\right]^{11}$. The two dimensional Laplace transform of the function 
$f\left(z_{1}, z_{2}\right)=f_{Z}(z)$ in two variables (denoted as random vector $Z$ ) is defined as:

$$
\mathcal{L}_{Z}\left(s_{1}, s_{2}\right)=\int_{\mathcal{D}_{1} \times \mathcal{D}_{2}} \exp \left(-s_{2} z_{2}-s_{1} z_{1}\right) f\left(z_{1}, z_{2}\right) d z_{1} d z_{2}
$$

where $\mathcal{D}_{i}$ refers the domain at which the probability density function $f_{Z_{i}}\left(z_{i}\right)$ of $Z_{i}$ is non-zero. ${ }^{19}$ Consequently, the one dimensional Laplace transform is defined as $\mathcal{L}_{Z_{1}}\left(s_{1}\right)=\int_{\mathcal{D}_{1}} \exp \left(-s_{1} z_{1}\right) f\left(z_{1}\right) d z_{1}$. Depending on the distributional assumptions regarding the frailties $\left(Z_{1}, Z_{2}\right)$, the Laplace transforms exhibit either closed-form expressions or should be approximated numerically.

\section{2 | Likelihood function for current status data}

Here, we focus on so-called bivariate CS data $\left(t_{1 j}, t_{2 j}, \delta_{1 j}, \delta_{2 j}\right)$ where $t_{i j}$ and $\delta_{i j}$ refer to the observation times and censoring indicators, i.e., $\delta_{i j}=I\left(T_{i j}^{*} \leq t_{i j}\right)$ are realizations of $\Delta_{i j}$, for event $i=1,2$ and individual/cluster $j=1, \ldots, n$. Parameter estimation for the proposed general frailty model was performed using maximum likelihood techniques. In case of current status data, the loglikelihood contribution for $\left(t_{1 j}, t_{2 j}, \delta_{1 j}, \delta_{2 j}\right)$ can be written as 20 :

$$
\begin{array}{r}
l_{j}\left(t_{1 j}, t_{2 j}, \delta_{1 j}, \delta_{2 j} \mid \boldsymbol{\theta}, \boldsymbol{\psi}\right)=\delta_{1 j} \delta_{2 j} \log \left[1-S_{1}\left(t_{1 j} \mid \boldsymbol{\theta}, \boldsymbol{\psi}\right)-S_{2}\left(t_{2 j} \mid \boldsymbol{\theta}, \boldsymbol{\psi}\right)+S\left(t_{1 j}, t_{2 j} \mid \boldsymbol{\theta}, \boldsymbol{\psi}\right)\right] \\
+\delta_{1 j}\left(1-\delta_{2 j}\right) \log \left[S_{2}\left(t_{2 j} \mid \boldsymbol{\theta}, \boldsymbol{\psi}\right)-S\left(t_{1 j}, t_{2 j} \mid \boldsymbol{\theta}, \boldsymbol{\psi}\right)\right] \\
+\left(1-\delta_{1 j}\right) \delta_{2 j} \log \left[S_{1}\left(t_{1 j} \mid \boldsymbol{\theta}, \boldsymbol{\psi}\right)-S\left(t_{1 j}, t_{2 j} \mid \boldsymbol{\theta}, \boldsymbol{\psi}\right)\right] \\
+\left(1-\delta_{1 j}\right)\left(1-\delta_{2 j}\right) \log \left[S\left(t_{1 j}, t_{2 j} \mid \boldsymbol{\theta}, \boldsymbol{\psi}\right)\right],
\end{array}
$$

in terms of the unconditional survival functions as introduced previously and explicitly spelling out the dependence on vectors of model parameters $\boldsymbol{\theta}$, and $\boldsymbol{\psi}$ associated with the baseline hazard functions and frailty distributions, respectively. The parameters included in the parameter vectors $\boldsymbol{\theta}$ and $\boldsymbol{\psi}$ differ from model to model depending on the selected copula and baseline hazard functions. Consequently, from this point onwards, the notation used will state this parameter configuration explicitly for each model discussed.

\section{3 | Bivariate frailty models}

The association between the frailty terms is reflected in the functional form of the joint survival function $S\left(t_{1}, t_{2} \mid \boldsymbol{\theta}, \boldsymbol{\psi}\right)$. The most simple and restrictive assumption is one of independent frailty terms $Z_{i}, i=1,2$. In the survival literature, this model is referred to as a univariate frailty model, implying that $S\left(t_{1}, t_{2} \mid \boldsymbol{\theta}, \boldsymbol{\psi}\right)=\boldsymbol{S}_{1}\left(t_{1} \mid \boldsymbol{\theta}_{1}\right) S_{2}\left(t_{2} \mid \boldsymbol{\theta}_{2}\right)$ and both events can be modeled separately, at least if no other model parameters are shared for the two processes (e.g., parameters related to the baseline hazard functions). However, in many settings the event times are dependent, hence, stressing the need for less restrictive models. To this end, many correlated frailty models have been proposed in the survival literature , for example, correlated gamma frailty models, correlated log-normal frailty models, correlated compound Poisson frailty models, to name a few. ${ }^{11}$ Hereunder, we discuss the attractive (additive) correlated gamma frailty model proposed by Yashin et al. ${ }^{16}$

The bivariate frailty distribution in the correlated gamma frailty model is constructed using independent components $Y_{i}$ with shape and inverse scale parameters equal to $k_{i}$ and 1, respectively, and denoted by $Y_{l} \sim \Gamma\left(k_{l}, 1\right)$ $\left(k_{I}>0, I=0,1,2\right)$, that is, $Z_{i}=\sigma_{i}^{2}\left(Y_{0}+Y_{i}\right)(i=1,2)$. In this 'variable-in-common' construction, $Y_{0}$ is the common component imposing a positive correlation between the frailties whereas $Y_{1}$ and $Y_{2}$ are infection-specific components. 
It is well established that bivariate correlated frailty models (with different but dependent frailties $Z_{1}$ and $Z_{2}$ ) with infinite means are not identifiable. ${ }^{14,21}$ Therefore constraining the means to be one and selecting parametric baseline hazards are necessary such that model parameters can be estimated. Imposing unit frailty means for identifiability, one can easily observe that the frailty variances are equal to $\sigma_{i}^{2}=\left(k_{0}+k_{i}\right)^{-1}$. Further, the Pearson correlation is given by $\rho=k_{0} / \sqrt{\left(k_{0}+k_{1}\right)\left(k_{0}+k_{2}\right)}$, leading to the constraint $0 \leq \rho \leq \min \left(\sigma_{1} \sigma_{2}^{-1}, \sigma_{2} \sigma_{1}^{-1}\right)$. The additive decomposition of the frailty terms in independent components leads to a tractable expression for the joint unconditional survival function in terms of the marginal survival functions:

$$
S\left(t_{1}, t_{2} \mid \boldsymbol{\theta}, \boldsymbol{\psi}\right)=\left[S_{1}\left(t_{1}\right)\right]^{1-\left(\sigma_{1} / \sigma_{2}\right) \rho}\left[S_{2}\left(t_{2}\right)\right]^{1-\left(\sigma_{2} / \sigma_{1}\right) \rho}\left[S_{1}\left(t_{1}\right)^{-\sigma_{1}^{2}}+S_{2}\left(t_{2}\right)^{-\sigma_{2}^{2}}-1\right]^{\rho /\left(\sigma_{1} \sigma_{2}\right)} .
$$

In order to avoid confusion with our general frailty models introduced later on, this model is referred to as the additive model throughout the remainder of the manuscript. Identifiability of this model is ensured by considering different parametric baseline hazard functions. Alternatively, a popular choice in survival analysis is the one of a shared or common frailty term for both events associated with the same subject. More specifically, $Z_{1}=Z_{2} \equiv Z$, thereby leading to $S\left(t_{1}, t_{2} \mid \boldsymbol{\theta}, \boldsymbol{\psi}\right)=\mathcal{L}\left[\Lambda_{10}\left(t_{1} \mid \boldsymbol{\theta}_{1}\right)+\Lambda_{20}\left(t_{2} \mid \boldsymbol{\theta}_{2}\right)\right]$. Note that the shared gamma frailty model is a special case of the aforementioned model in which $Y_{1}$ and $Y_{2}$ have degenerate distributions at 0 and $\rho$ tends to 1 if $k_{l} \rightarrow 0, I=1,2$. Although the shared frailty model is a very popular choice, it is quite restrictive in terms of the implied correlation structure.

\section{4 | Copula functions}

In this section, we give a brief introduction to bivariate copula functions. A function $C:[0,1] \times[0,1] \rightarrow[0,1]$ is called a copula function when it has the following properties:

(i) For every $u, v \in[0,1]: C(u, 0)=C(0, v)=0$ and $C(u, 1)=u$ and $C(1, v)=v$.

(ii) For every $u_{1}, u_{2}, v_{1}, v_{2} \in[0,1]$ such that $u_{1} \leq u_{2}$ and $v_{1} \leq v_{2}: C\left(u_{2}, v_{2}\right)-C\left(u_{2}, v_{1}\right)-C\left(u_{1}, v_{2}\right)+C\left(u_{1}, v_{1}\right) \geq 0$.

From these properties, we note that the bivariate copula function $C$ is the joint (cumulative) distribution function of a two dimensional random vector $(U, V)$ on the unit cube $[0,1] \times[0,1]$ with uniform margins. Furthermore, from Sklar's theorem ${ }^{22}$, it follows that for any continuous random vector $(X, Y)$ with joint distribution $H$ and respective marginals $F$ and $G$, there exists a unique copula function $C$ such that for all $x, y \in R$ :

$$
H(x, y)=C[F(x), G(y)]
$$

Several families of copulae have been described in the literature. Among them, Gaussian and Archimedean copulae are quite popular and widely used in real-life applications. For example, the Gaussian copula was used in geostatistical interpolation ${ }^{23}$, marketing research ${ }^{24}$, and in copula-based regression models for a bivariate mixed outcome ${ }^{25,26}$, among other areas. The Archimedean copulae family was used in insurance analysis ${ }^{27}$, in bivariate survival data ${ }^{4}$, and in insurance experience rating ${ }^{28}$, to name a few. 


\subsection{Our proposed general frailty model}

Let us denote the joint distribution of the frailty vector $\left(Z_{1}, Z_{2}\right)$ by:

$$
F\left(z_{1}, z_{2}\right)=P\left(Z_{1} \leq z_{1}, Z_{2} \leq z_{2}\right)=C\left[F_{1}\left(z_{1}\right), F_{2}\left(z_{2}\right)\right]=C\left(u_{1}, u_{2}\right)
$$

in which $u_{1}=F_{1}\left(z_{1}\right)=P\left(Z_{1} \leq z_{1}\right)$ and $u_{2}=F_{2}\left(z_{2}\right)=P\left(Z_{2} \leq z_{2}\right)$ are the marginal cumulative distribution functions of the frailty variables $Z_{1}$ and $Z_{2}$, respectively, and $C\left(u_{1}, u_{2}\right)$ is a copula function defining the joint distribution of the frailty terms. By construction, we have $0 \leq u_{1}, u_{2} \leq 1$. Furthermore, the copula function $C$ completely determines the association between the frailty terms $Z_{1}$ and $Z_{2}$.

In general, we can write the joint survival function of the two event times as:

$$
\begin{aligned}
S\left(t_{1}, t_{2} \mid \boldsymbol{\theta}, \boldsymbol{\psi}\right) & =P\left(T_{1}>t_{1}, T_{2}>t_{2}\right) \\
& =\int_{0}^{\infty} \int_{0}^{\infty} \exp \left[-z_{1} \Lambda_{10}\left(t_{1} \mid \boldsymbol{\theta}_{1}\right)-z_{2} \Lambda_{20}\left(t_{2} \mid \boldsymbol{\theta}_{2}\right)\right] \partial^{2} F\left(z_{1}, z_{2} \mid \boldsymbol{\psi}\right) \\
& =\int_{0}^{\infty} \int_{0}^{\infty} \exp \left[-z_{1} \Lambda_{10}\left(t_{1} \mid \boldsymbol{\theta}_{1}\right)-z_{2} \Lambda_{20}\left(t_{2} \mid \boldsymbol{\theta}_{2}\right)\right] f\left(z_{1}, z_{2} \mid \boldsymbol{\psi}\right) \mathrm{d} z_{1} \mathrm{~d} z_{2} \\
& =\int_{0}^{1} \int_{0}^{1} \exp \left[-F_{1}^{-1}\left(u_{1}\right) \Lambda_{10}\left(t_{1}\right)-F_{2}^{-1}\left(u_{2}\right) \Lambda_{20}\left(t_{2}\right)\right] c\left(u_{1}, u_{2}\right) \mathrm{d} u_{1} \mathrm{~d} u_{2},
\end{aligned}
$$

where $f\left(z_{1}, z_{2}\right)$ is the joint density function of $\left(Z_{1}, Z_{2}\right), c\left(u_{1}, u_{2}\right)$ is the copula density function and $F^{-1}$ denotes the quantile function of a continuous distribution $F$. The general copula formulation of the joint distribution $F\left(z_{1}, z_{2}\right)$ allows for a separate treatment of the association between the frailty terms (given by the selected copula function) and the amount of unobserved heterogeneity (given by the marginal frailty distributions).

We demonstrate now that by considering different marginal distributions for the frailty terms $Z_{i}$ in combination with different copula functions $C\left(u_{1}, u_{2}\right)$, we obtain existing frailty models. For example, let $C\left(u_{1}, u_{2}\right)=u_{1}$. $u_{2}$. This corresponds to the independent copula function. Then our general model is equivalent to an independent frailty model. As a second example, let $C\left(u_{1}, u_{2}\right)=\min \left(u_{1}, u_{2}\right)$ and consider the same marginal gamma distribution for the frailty terms $Z_{1}$ and $Z_{2}$. In this case, our general frailty model reduces to a shared gamma frailty model with perfect positive correlation between the frailty variables. In a third example, we assume that the random frailty variables $Z_{i}=\sigma_{i}^{2}\left(Y_{0}+Y_{i}\right)$ where $i=1,2$ with $Y_{i}$ independent and $Y_{i} \sim \Gamma\left(k_{i}, 1\right)$. If we take a copula function given by $C\left(u_{1}, u_{2}\right)=P\left(Y_{0}+Y_{1} \leq F_{1}^{-1}\left(u_{1}\right), Y_{0}+Y_{2} \leq F_{2}^{-1}\left(u_{1}\right)\right)$ with $F_{i}^{-1}$ the quantile function of $Y_{0}+Y_{i} \sim \Gamma\left(k_{0}+k_{i}, 1\right), i=1,2$, the proposed general frailty model reduces to the well-known additive correlated gamma frailty model proposed by Yashin et $\mathrm{al}^{16}$. The reader is referred to Web Appendix B of the Supplementary Materials for the mathematical derivations showing that these traditional frailty models are special cases of the general frailty model presented above.

Throughout this manuscript, $Z_{i}$ is assumed to follow a gamma frailty distribution with shape and inverse scale parameters equal to $\alpha_{i}$, i.e., $Z_{i} \sim \Gamma\left(\alpha_{i}, \alpha_{i}\right)$, implying unit mean and frailty variance $\sigma_{i}^{2}=1 / \alpha_{i}$. The gamma distribution was chosen due to its mathematical convenience in the sense that its Laplace transform has a closed form. Moreover, the gamma distribution is the only continuous distribution that does not impose a time-varying association between bivariate event times as expressed in terms of the cross-ratio function. ${ }^{17}$ Obviously, other frailty distributions could be incorporated as well. Hereunder, we introduce some different choices with regard to the copula density $c\left(u_{1}, u_{2}\right)$ in order to impose a more flexible correlation structure between the frailties $Z_{1}$ and $Z_{2}$. In case a Gaussian copula is chosen, we refer to the model as a Gaussian copula-frailty model whereas if a Frank copula is used, the model is referred to a Frank copula-frailty model, etc. 


\section{Gaussian copula-frailty model}

The bivariate Gaussian copula function takes the form $C\left(u_{1}, u_{2} ; \rho\right)=\Phi_{2}\left[\Phi^{-1}\left(u_{1}\right), \Phi^{-1}\left(u_{2}\right)\right]$ with Pearson productmoment correlation coefficient $-1 \leq \rho \leq 1$ among the marginal random variables. Here, $\Phi_{2}(\cdot)$ denotes the quantile function of a bivariate normal distribution with correlation $\rho$ and $\Phi(\cdot)$ denotes the quantile function of a standard normal distribution. The bivariate Gaussian copula density is given by ${ }^{29}$ :

$$
c\left(u_{1}, u_{2} ; \rho\right)=\frac{1}{\sqrt{1-\rho^{2}}} \exp \left(\frac{2 \rho \Phi^{-1}\left(u_{1}\right) \Phi^{-1}\left(u_{2}\right)-\rho^{2}\left\{\left[\Phi^{-1}\left(u_{1}\right)\right]^{2}+\left[\Phi^{-1}\left(u_{2}\right)\right]^{2}\right\}}{2\left(1-\rho^{2}\right)}\right),
$$

When $\rho \rightarrow 1$, the Gaussian copula approaches the upper Fréchet-Hoeffding bound indicating comonotonicity of the random variables $Z_{1}$ and $Z_{2}{ }^{29}$ In that case, the Gaussian copula-frailty model approaches a shared frailty model as $F\left(z_{1}, z_{2}\right)=\min _{i}\left[F_{i}\left(z_{i}\right)\right]$ when $\rho \rightarrow 1$. When $\rho=0$, the Gaussian copula function becomes the independence copula, and the copula-frailty model is equivalent to a univariate frailty model with independent frailty terms $Z_{1}$ and $Z_{2}$.

\section{Frank copula-frailty model}

A Frank copula-frailty model is formulated based on the Frank copula which is a member of the Archimedean copula family. In general, the Archimedean copula family is one of the most popular copula families to model bivariate survival data. A bivariate Archimedean copula can be represented by:

$$
C\left(u_{1}, u_{2} ; \vartheta\right)=\phi_{\vartheta}\left[\phi_{\vartheta}^{-1}\left(u_{1}\right)+\phi_{\vartheta}^{-1}\left(u_{2}\right)\right]
$$

where $0 \leq \phi_{\vartheta}(\cdot) \leq 1, \phi_{\vartheta}(0)=1, \phi_{\vartheta}^{\prime}(\cdot)<0$, and $\phi_{\vartheta}^{\prime \prime}(\cdot)>0$ (see, for example, ${ }^{22}$ ).

Three important members of the Archimedean copula family are the Clayton, Gumbel and Frank copula. ${ }^{4}$ While the Clayton and Gumbel copulae only capture non-negative dependence, the Frank copula offers the possibility to represent both negative and positive correlation. ${ }^{22}$ More specifically, the bivariate Frank copula function takes the form:

$$
C\left(u_{1}, u_{2} ; \vartheta\right)=\frac{1}{\log (\vartheta)} \log \left[1+\frac{\left(\vartheta^{u_{1}}-1\right)\left(\vartheta^{u_{2}}-1\right)}{\vartheta-1}\right],
$$

where $\vartheta>0$ and $\vartheta \neq 1$. When $\vartheta>1$ there exists a negative association between the random variables. ${ }^{4}$ The bivariate Frank copula density is given by:

$$
c\left(u_{1}, u_{2}\right)=\frac{(\vartheta-1) \vartheta^{u_{1}+u_{2}} \log (\vartheta)}{(\vartheta-1)+\left(\vartheta^{u_{1}}-1\right)\left(\vartheta^{u_{2}}-1\right)},
$$

which can be introduced in equation (2) to obtain an expression for the joint survival function $S\left(t_{1}, t_{2}\right)$. For more details on the derivation of $c\left(u_{1}, u_{2}\right)$, the reader is referred to Web Appendix A of the Supplementary Materials.

\section{Clayton copula-frailty model}

The bivariate Clayton copula function takes the form:

$$
C\left(u_{1}, u_{2} ; \vartheta\right)=\left(u_{1}^{-\vartheta}+u_{2}^{\vartheta}-1\right)^{-\frac{1}{\vartheta}}
$$

where $0<\vartheta<+\infty$. When $\vartheta \rightarrow+\infty$, the Clayton copula converges to the monotonicity copula with the correlation approaching 1 , and when $\vartheta \rightarrow 0$, the Clayton copula converges to the independent copula with the correlation ap- 
proaching zero. ${ }^{22}$ As stated before, the Clayton copula function can capture only positive association. The Clayton copula density is given by ${ }^{30}$ :

$$
c\left(u_{1}, u_{2}\right)=(1+\vartheta)\left(u_{1} u_{2}\right)^{-1-\vartheta}\left(u_{1}^{-\vartheta}+u_{2}^{-\vartheta}-1\right)^{-\frac{1}{\vartheta}-2},
$$

Again, this copula density function can be plugged in equation (2) to obtain the corresponding bivariate joint survival function $S\left(t_{1}, t_{2} \mid \boldsymbol{\theta}, \boldsymbol{\psi}\right)$.

\section{Gumbel copula-frailty model}

The bivariate Gumbel copula function takes the form:

$$
C\left(u_{1}, u_{2} ; \vartheta\right)=\exp \left[-\left(t_{1}+t_{2}\right) \frac{1}{\vartheta}\right]
$$

where $t_{1}=\left[-\log \left(u_{1}\right)\right]^{\vartheta}, t_{2}=\left[-\log \left(u_{2}\right)^{\vartheta}\right], \vartheta \geq 1$. When $\vartheta \rightarrow+\infty$, the Gumbel copula converges to the comonotonic copula with the correlation approaching 1 , and when $\vartheta \rightarrow 1$, the Gumbel copula converges to independent copula with the correlation approaching zero. ${ }^{22}$ Similarly to the Clayton copula, the Gumbel copula function can not account for a negative association. The bivariate Gumbel copula density is given by ${ }^{30}$ :

$$
c\left(u_{1}, u_{2}\right)=C\left(u_{1}, u_{2} ; \vartheta\right) \frac{1}{u_{1} u_{2}}\left(t_{1}+t_{2}\right)^{-2+2 / \vartheta}\left[\log \left(u_{1}\right) \log \left(u_{2}\right)\right]^{\vartheta-1}\left[1+(\vartheta-1)\left(t_{1}+t_{2}\right)^{-\frac{1}{\vartheta}}\right],
$$

This copula density function can be used in equation (2) to obtain an expression for $\mathcal{S}\left(t_{1}, t_{2} \mid \boldsymbol{\theta}, \boldsymbol{\psi}\right)$.

\subsection{1 | Association measures}

In order to describe the association between the frailties and compare the strength thereof across the different models, one can rely on one of the most familiar dependence measures, namely the Pearson product-moment correlation. However, the correlation coefficient is a measure of linear association between $Z_{1}$ and $Z_{2}$, an assumption which could be violated. Alternatively, Kendall's tau is a rank correlation measure which can be applied in case the association is non-linear and it only depends on the choice of the copula function $C$. The expression for Kendall's tau in case of the Frank copula is given by: $\tau_{F}=1+4\left[D_{1}(\theta)-1\right] / \theta$, where $\theta=-\log (\vartheta)$ and $D_{k}(\gamma)$ is the Debye function given by:

$$
D_{k}(\gamma)=\frac{k}{\gamma^{k}} \int_{0}^{\gamma} \frac{t^{k}}{\exp (t)-1} \mathrm{~d} t
$$

where $k=1,2 .{ }^{31}$ In case of a Clayton copula, the Kendall's tau is simply given by $\tau_{\text {Clayton }}=\frac{\vartheta}{\vartheta+2}$, and $\tau_{\text {Gumbel }}=1-\frac{1}{\vartheta}$ for a Gumbel copula. ${ }^{22}$ If we use a Gaussian copula function, Kendall's tau is given by $\tau_{G}=2 \arcsin (\rho) / \pi .{ }^{22}$ The variance of Kendall's tau can be calculated based on the variance of $\theta$ or $\rho$ using the Delta method.

For the additive model, the calculation of Kendall's tau is less straightforward since a closed-form expression is absent. Nonetheless, a simulation-based empirical estimate of Kendall's tau can be calculated by looking at the concordance score of pairs of bivariate observations $\left[\left(Z_{1 j}, Z_{1 k}\right),\left(Z_{2 j}, Z_{2 k}\right)\right]$ generated based on the estimated frailty distributions. Hence, Kendall's tau can be obtained as the difference of the probability of concordance and discordance ${ }^{15,32}$, 
that is:

$$
\tau_{S}=P\left[\left(Z_{1 j}-Z_{1 k}\right)\left(Z_{2 j}-Z_{2 k}\right)>0\right]-P\left[\left(Z_{1 j}-Z_{1 k}\right)\left(Z_{2 j}-Z_{2 k}\right)<0\right]
$$

Since the estimate of $\tau_{S}$ is made over several simulations, the simulation-based standard error can be calculated as well.

\section{4 | SIMULATION STUDY}

In order to gain deeper insights in the performance of the general frailty models introduced in Section 3.5 when analyzing bivariate CS data, we performed a simulation study. More specifically, we studied the impact of misspecification of the bivariate frailty distribution, and the effect of restrictive bounds on the implied association structure. In general, we generated bivariate TTE data according to the following three steps. First, frailty terms $\left(z_{1 j}, z_{2 j}\right)$ were simulated from the presumed bivariate frailty distribution. After generating values $z_{i j}(i=1,2, j=1, \ldots, n)$, event times $t_{i j}^{*}$ were generated by solving the equation $s_{i}\left(t_{i j}^{*} \mid z_{i j}\right)=\exp \left[-z_{i j} \wedge_{i 0}\left(t_{i j}^{*}\right)\right]=u_{i j}$, where $u_{i j}$ was randomly generated from a standard uniform distribution and $\Lambda_{i 0}\left(t_{i j}^{*}\right)$ is a specific parametric cumulative baseline hazard function. Next, independent univariate monitoring times were generated from a uniform distribution, i.e., $T_{1 j}=T_{2 j} \equiv T_{j} \sim U(0,100)$. Consequently, censoring indicators $\delta_{i j}$ indicated whether or not the simulated event times $t_{i j}^{*}$ preceded the monitoring times $T_{j}$.

Bivariate gamma frailties $\left(z_{1 j}, z_{2 j}\right)$ were generated based on three different models:

(i) Additive correlated gamma frailty model: additive components $y_{l j}(I=0,1,2)$ were obtained from $\Gamma\left(k_{l}, 1\right)$ distributions. Consequently, $z_{i j}=\sigma_{i}^{2}\left(y_{0 j}+y_{i j}\right)(i=1,2)$ with sample size $n(j=1,2, \ldots, n)$.

(ii) Gaussian copula-frailty model: $\left(z_{1 j}, z_{2 j}\right)$ were generated from a bivariate Gaussian copula with correlation parameter $\rho$ and marginal gamma distributions $\Gamma_{1}\left(\alpha_{1}, \alpha_{1}\right)$ and $\Gamma_{2}\left(\alpha_{2}, \alpha_{2}\right)$ for $Z_{1}$ and $Z_{2}$, respectively, with sample size $n$ $(j=1,2, \ldots, n)$.

(iii) Frank copula-frailty model: $\left(z_{1 j}, z_{2 j}\right)$ were generated from a bivariate Frank copula with association parameter $\vartheta$ and marginal gamma distributions $\Gamma_{1}\left(\alpha_{1}, \alpha_{1}\right)$ and $\Gamma_{2}\left(\alpha_{2}, \alpha_{2}\right)$ for $Z_{1}$ and $Z_{2}$, respectively, with sample size $n$ $(j=1,2, \ldots, n)$.

One of the disadvantages of the additive correlated gamma frailty model introduced by Yashin et al. ${ }^{16}$ is the restriction of a non-negative correlation between the frailty terms $Z_{1}$ and $Z_{2}$. On top of that, the correlation is also restricted above by the ratio of the frailty standard deviations as indicated in Section 3.3. Hereunder, we mainly focus on simulating CS data arising from choices of the association between the frailty terms which are implausible for the additive model to capture (e.g., negative association). We examine the performance of the novel general frailty models in this case as well as the impact of incorrectly fitting the additive model.

In the analysis of survival data, certain parametric distributions for the baseline hazard function have been employed repeatedly, for example, Exponential, Gompertz and Weibull hazards. In our simulation setting, Gompertz baseline hazard functions were chosen since this baseline hazard function is commonly used in the context of analysing epidemiological and infectious disease data. ${ }^{11}$ For example, Hens et al. ${ }^{15}$ used Gompertz baseline hazard functions to fit a correlated gamma frailty model to the hepatitis A virus - hepatitis B virus bivariate current status data. However, other parametric choices for the baseline hazard function could be used instead. 


\section{1 | Simulation from the additive correlated gamma frailty model}

First of all, we generated CS data from the additive correlated gamma frailty model (with a sample size of $n=3791$ for each data set). We analyzed 300 simulated datasets using the additive model, the Frank copula-frailty model, the Gaussian copula-frailty model, the Clayton copula-frailty, and the Gumbel copula-frailty model. The results show that our proposed copula-frailty models performed equally well as compared to the estimates obtained when fitting the correct underlying model (see Table 1).

[Table 1 is about here ]

\section{2 | Simulation from a Gaussian copula-frailty model}

In this section, we consider simulation results under two different scenarios for the Gaussian copula-frailty model, namely, with a negative correlation close to zero $(\rho=-0.1)$ and a negative correlation close to $-1(\rho=-0.9)$. Table 2 shows the mean estimate, empirical standard error (SE), mean squared error (MSE), and median estimate for all parameters based on 300 simulation datasets with sample size $n=3791$. This large sample size is in line with the sample size in the serological data applications. Results of a simulation setting with a smaller sample size $(n=500)$ are provided in Web Appendix C of the Supplementary Materials.

As expected, when $\rho<0$, the additive gamma frailty model was only able to estimate the correlation coefficient to be fairly close to zero whereas the Gaussian copula-frailty model provided a good estimate for the correlation parameter. In order for the additive model to force $\rho$ to zero, $\alpha_{1}$ and $\alpha_{2}$ were estimated to be extremely large (with large standard error estimates) in some simulation runs giving rise to departures of mean estimates from the true values and median estimates that are close thereto. It is worth noticing that the mean estimates for the variance parameters related to the two frailty terms in our proposed copula-frailty models differed substantially from the true underlying values. It has been shown before that, in case of CS data, the estimators for the model parameters are consistent (hence asymptotically unbiased), but that the finite sample performance is affected by the information loss when turning from uncensored TTE data to CS data. More specifically, Hens et al. ${ }^{15}$ demonstrated this behaviour using various simulation settings in which the authors analyzed and compared the performance of correlated gamma frailty models when applied to uncensored, right-censored and CS data. Consequently, we expect to see the same behaviour here for our proposed copula-frailty models. On the other hand, the median estimates were found to be very close to the true values for the baseline hazard parameters (in case of the copula-frailty models and additive gamma frailty model), as well as for the variance parameters related to the two frailty terms (in our proposed copulafrailty models) implying a skewed finite sample distribution for the corresponding estimators. In approximately $95 \%$ of the simulated datasets (with $\rho=-0.1$ ), the Akaike Information Criterion (AIC) ${ }^{33}$ was lower for the correct model compared to the one for the additive counterpart. In the case of $\rho=-0.9$, all AIC-values obtained when applying the Gaussian copula-frailty model to the generated datasets were smaller than the ones obtained using the additive model.

Moreover, we used the Frank copula-frailty model to analyze data simulated from a Gaussian copula-frailty model. Generally, the Frank copula-frailty model performed very well in comparison with the additive gamma frailty model and did reasonably well compared with the correctly-specified Gaussian copula-frailty model. However, the estimates of Kendall's tau when using the Frank copula-frailty model were generally lower than those obtained from the Gaussian copula-frailty model. Moreover, in terms of AIC, there are only $3 \%$, and $0 \%$ cases (respectively for the settings of $\rho=-0.1$, and $\rho=-0.9$ ) in which a correctly-specified Gaussian copula-frailty model gave a higher AIC value than the corresponding value in case of a Frank copula-frailty model. In most of the runs where the AIC value of the Gaussian 
copula-frailty model was higher than that of the Frank copula-frailty model, the difference in AIC values was smaller than one. Hence, model selection based on AIC is reasonable.

We did not use the Clayton copula-frailty, and the Gumbel copula-frailty model to analyze these simulated data since these two copula-frailty models could not capture a negative association between the two event times.

[Table 2 is about here ]

\section{3 | Simulation from a Frank copula-frailty model}

Two values for the association parameter $\vartheta$ were selected, namely $\vartheta=1 \mathrm{E} 4$ (corresponding to a negative association between frailty terms with Kendall's tau equal to $\tau_{F}=-0.643$ ) and $\theta=8.2$ which leads to a small negative association quantified as $\tau_{F}=-0.224$. Table 3 shows the mean estimate, empirical SE, MSE, and median estimate for the model parameters based on 300 simulation datasets with a sample size of $n=3791$. Again, results of a simulation setting with a smaller sample size are presented in Web Appendix $C$ of the Supplementary Materials.

As expected, when $\tau_{F}<0$, only the Frank copula-frailty model could give a close estimate of the $\tau$ parameter, whereas the corresponding $\tau$ parameter in the additive model was estimated to be a very small positive number. The estimates of $\alpha_{1}$ and $\alpha_{2}$ were again large (with large standard errors) implying small frailty variances. Moreover, AICvalues for the additive models were found to be bigger (in 300 simulated datasets) compared to the ones for the Frank copula frailty models in both cases. Hence, identification of the correct model based on AIC is possible.

Additionally, we employed the Gaussian copula-frailty model to analyze data generated from a Frank copula-frailty model. Generally speaking, the Gaussian copula-frailty model performed equally well as the correctly-specified Frank copula-frailty model. We also did not use a Clayton, and a Gumbel copula-frailty model to analyze these simulated data since these two models could not capture a negative association.

[Table 3 is about here]

\subsection{Additional simulation results}

We provided many additional simulation results in Web Appendix C of the Supplementary Materials. When the association parameter exceeds the restrictive bound in the additive model, the general frailty models provide a useful alternative to describe the association structure appropriately. In contrast, the additive model is unable to capture the association without forcing the frailty variances to be close to each other in order to enable the correlation parameter to be estimated large enough. Hence, misspecification of the additive structure leads to biased estimates of the frailty variances.

We also reported the results under simulation settings with other baseline hazard functions, for example, exponential and Weibull hazard functions. Generally, we could draw the same conclusion as in the scenarios with data generating using Gompertz baseline hazard functions. We invite interested readers to go through the Supplementary Materials for more detailed results.

\section{5 | CASE STUDIES}

As mentioned previously, we applied the novel copula-frailty methodology to bivariate CS data on (1) VZV and PVB19 infections and (2) VZV and HAV infections. In all settings, marginal gamma frailty distributions were considered, and different parametric baseline hazard functions were applied for the infections at hand, i.e., exponential, Weibull and 
Gompertz baseline hazards. Model selection was performed based on $\mathrm{AlC}^{33}$. In these analyses, the monitoring times $t_{i j}$ were represented by the individual's age at data collection, and univariate monitoring times $t_{i j} \equiv t_{j}, j=1, \ldots, n$ were present.

\section{1 | Varicella zoster virus and parvovirus B19}

There were 2975 individuals with complete infection statuses for VZV and PVB19. First, we fit the additive correlated gamma frailty model using different baseline hazard functions (exponential, Weibull and Gompertz). Since the model with Weibull baseline hazard functions $\left(\lambda_{i 0}\left(t_{i j}\right)=\rho_{i} \zeta_{i} t_{i j}^{\rho_{i}-1}, i=1,2\right)$ performed best in terms of AIC, we show results of fitting different copula-frailty models with Weibull baseline hazards. Table 4 shows the parameter estimates using the copula-frailty models and additive correlated gamma frailty model. Based on AIC, the general frailty models outperformed the existing additive correlated gamma frailty model. More specifically, the Gumbel copula-frailty model performed best (AIC $=4491.5580$ ), followed closely by the Frank copula-frailty, the Gaussian copula-frailty, and the Clayton copula-frailty model (AIC $=4492.7110,4494.0290$ and 4497.9710, respectively). Furthermore, parameter and standard error estimates for the aforementioned models were very close to each other.

[Table 4 is about here]

The association between the infection-specific frailties across the different models is expressed in terms of Kendall's tau with values $\hat{\tau}=0.2000$ (0.0535), $\hat{\tau}=0.1841$ (0.0530), $\hat{\tau}=0.1481(0.0530), \hat{\tau}=0.0727(0.0303)$, and $\hat{\tau}=$ 0.1492 ( 0.0121$)$ for the Gumbel copula-frailty, Frank copula-frailty, Gaussian copula-frailty, Clayton copula-frailty and the additive correlated gamma frailty model, respectively. It could be seen that Kendall's tau estimates are quite similar between the Gumbel copula-frailty, and Frank copula-frailty, and between the Gaussian copula-frailty and the additive gamma frailty model. However, the estimate obtained from the Clayton copula-frailty model is smaller in comparison with the results using the other four models. This discrepancy can be explained by the different association structures imposed by the different copula functions.

Figure 1 shows the scatter plots of two frailty terms $Z_{1}, Z_{2}$ produced under different fitted models. The plot reflects different association structures introduced by different models. Mimicking the work of Munda et al. ${ }^{34}$, one might predict the frailty terms in our copula-frailty model. This would allow us to compare these empirical estimates with the frailty terms under the fitted models to develop a selection procedure for the copula function in our model. However, at this moment, this would lead us away from the focus of the current manuscript. Thus, we consider postponing this research for a follow-up paper.

[Figure 1 is about here]

The fitted joint probabilities of VZV and PVB19 infections using estimates from the Gumbel copula-frailty model (best model fit in terms of AIC) and from the Frank copula-frailty model ("second best" model fit in terms of AIC) are shown in Figure 2. The figure indicates a good fit to the data using the models at hand. There is only a small difference in the fitted joint probabilities of the two diseases when using the Gumbel copula-frailty model and the Frank copula-frailty model, even with the "worst" additive gamma frailty model (not shown in Figure 2). In the future, if there is a dataset which possibly reveals a negative association between event times, we might observe a big difference between, for example, a fitted Frank copula-frailty model (can capture a negative association), and a fitted additive gamma frailty model (can capture only positive association).

[Figure 2 is about here] 


\section{2 | Varicella zoster virus and hepatitis A}

A total of 3256 individuals had infection statuses for both VZV and HAV infections. First, we fit the additive correlated gamma frailty model using different baseline hazard functions (exponential, Weibull and Gompertz). Since models with Weibull baseline hazard functions performed best regarding AIC, we fitted different copula-frailty models using these parametric baseline hazard functions.

In order to describe the observed seroprevalence of HAV adequately, we use models including a piecewise Weibull hazard function with a single cut-off point at age 30 based on a graphical inspection of the profile (see Web Appendix C.2 of the Supplementary Materials). Hence, the baseline hazard function for HAV is given by:

$$
\lambda_{20}\left(t_{j}\right)= \begin{cases}\zeta_{21} \varrho_{21} t_{j}^{\rho_{21}-1} & \text { if } t_{j} \leq 30 \\ \zeta_{22} \varrho_{22} t_{j}^{\rho_{22}-1} & \text { if } t_{j}>30\end{cases}
$$

For VZV, a Weibull hazard function was considered $\lambda_{10}\left(t_{j}\right)=\zeta_{1} \varrho_{1} t_{j}^{\rho_{1}-1}$. In Table 5, the parameter estimates of the three models are presented. Based on AIC, the copula-frailty models outperformed the existing additive correlated gamma frailty model. It is of note that, some of the parameters in our model are non-negative (e.g., variance parameters and some or all of the baseline hazard parameters depending on the choice of baseline hazard functions). Hence, constructing a symmetric $95 \%$ Wald-based confidence interval for these parameters based on the point estimates and estimated standard errors, which is equivalent to performing a two-sided Wald test, would be inappropriate. A graphical exploration of the model fit is displayed in the Web Appendix C.3.1 of the Supplementary Materials.

The estimate of $\alpha_{2}$ was found to be large in several of the fitted models, thereby implying a small variance for the frailty term $Z_{2}$. Here, this finding implies that individuals do not differ a lot in their (age-dependent) risk of acquiring hepatitis A. Under a general setting, when the variance of $Z_{2}$ tends to zero, the marginal survival function $S_{2}(t)$ approaches the exponential function $\exp \left[-\Lambda_{20}(t)\right]$, providing evidence of the absence of individual heterogeneity in the acquisition of the second infection. Moreover, in the limit, the infection times will behave as independent random variables (a proof thereof can be found in Web Appendix B.4 in the Supplementary Materials) which prevents a (reliable) estimation of the association parameter in the copula-frailty models due to unidentifiability.

[Table 5 is about here]

The large standard error estimates for Kendall's tau presented in Table 5 were obtained by using the delta method, for which the first order approximation of the Taylor series expansion could potentially be invalid thereby overestimating the standard errors. On the other hand, bootstrap-based 95\% percentile confidence intervals of Kendall's tau (relying on a nonparametric bootstrap approach) given by [0.3525, 0.3915], [0.0411, 0.0849], [0.1234, 0.1640], and $[0.1118,0.1518]$ for the Frank, Clayton, Gumbel, and Gaussian copula-frailty model, respectively, suggest a clear deviation from independence. Moreover, the independence model provided a worse fit to the data in terms of AIC (AIC $=4571.643$ ) as compared to the copula-frailty models.

To better understand the dynamics of these two diseases, we illustrated fitted baseline hazard functions of the two diseases in Figure 3. It could be seen that the force of infection (baseline hazard) of VZV increases monotonically with ages whereas for HAV, the force of infection first decreases from age one till age 30, and then for those older than 30 , the force of infection increases monotonically with ages.

[Figure 3 is about here] 


\section{6 | DISCUSSION AND CONCLUSION}

In this paper, we introduced a (copula-)frailty model in which a copula function is considered to describe the association between the different frailty variables. Within the research field of multivariate TTE data, an alternative approach to estimate the association between event times is using copula models. More specifically, the focus of copula models lies in describing the association between event times directly utilizing a copula function instead of indirectly, as we do in this paper, through imposing latent frailty variables representing individual heterogeneity in experiencing the events under consideration. In this indirect approach, the (bivariate) copula function is used to induce association between the frailty variables, rather than the event times.

Our novel general frailty model is appealing in the sense that any restrictions on the imposed correlation between event-specific frailty variables which are inevitable in case of a 'variable-in-common' structure are easily avoided. Furthermore, various bivariate frailty distributions can be constructed allowing for different tail dependencies and a negative association among frailties. On top of that, in case of misspecification, the general frailty models tend to provide parameter estimates which are close to the true values as we have observed through simulation from an additive correlated gamma frailty model. In the future, we want to study in more detail how the association between the time to event variables is influenced by the choice of the copula function, or equivalently, the joint distribution that is imposed for the event-specific frailty variables. For example, if bivariate CS data are generated from a Gaussian copula-frailty model (with some baseline hazard functions and specific correlation parameters corresponding to a specific value of Kendall's Tau), and we use other copula-frailty models to analyze these data, how would the estimated Kendall's Taus values be different from the "true" value.

Our proposed copula-frailty model made use of different copula functions which behave differently with respect to their tail dependencies. The Gaussian copula has no tail dependence, while the Frank copula has an equal lower and upper tail dependence. The Clayton copula has only a lower tail dependence while the Gumbel copula has only an upper tail dependence. At this moment, we have not explored whether we could use these differences in tail dependence as a formal or graphical tool to choose an appropriate copula function.

Throughout this manuscript, we focused on gamma distributed frailty terms, albeit other frailty distributions could be considered without additional derivations. Note, however, that the attractiveness of the gamma frailty distribution is partly due to the mathematical tractability of its Laplace transform. Hence, choosing another univariate frailty distribution without a closed-form expression for the Laplace transform increases the computational burden considerably. Furthermore, as we noted previously, the gamma distribution is the only continuous distribution not imposing a timevarying association between event (infection) times as can be expressed in terms of the cross-ratio function which was introduced by Glidden ${ }^{17}$. Hence, the gamma distribution can be seen as a sensible starting point for exploring the dependence structure in paired serological data. An interesting avenue for further research is to explore other frailty distributions, e.g., members of the PVF family of which the gamma distribution is a special case. ${ }^{11}$ The proposed methodology can be readily extended to do so. For reasons of identifiability, we require within this manuscript the specification of parametric baseline hazard functions. In the data applications considered here, we explored various choices thereof and selected the most appropriate event-specific parametric functions, within the set of candidates, based on Akaike's Information Criterion which is a common strategy in survival analysis. Identifiability of the proposed models is assessed through simulations, and a formal assessment of identifiability is considered beyond the scope of this manuscript.

The current manuscript focuses on the application of the proposed method to bivariate current status data. However, one can extend the method manageably in two directions. First of all, the method can be modified to analyze higher dimensional data, for example, trivariate or quadrivariate current status data. For the Gaussian copula-frailty 
model, the extension is straightforward by using a trivariate or quadrivariate gaussian copula function. However, one might need to impose a restricted variance-covariance structure so that the model is identifiable. In case of an Archimedian copula-frailty model, the extension to a higher dimensional problem is not straightforward but can be done using, for example, the pair-copula construction. ${ }^{35-37} \mathrm{~A}$ common problem when analyzing high dimensional data is that the computational aspect might become challenging. This opens a thought-provoking research avenue in the future. Secondly, although we have illustrated the novel general frailty methodology using two serological data applications entailing bivariate current status data, extensions towards other censoring types are straightforward (in line with the work by, for example, ${ }^{15}$ ).

The main application of our proposed copula-frailty model is to analyze (bivariate) current status data which resulted from a parallel design in which we assume several different lifetimes for each individual. As far as we have investigated, this model is also suited for data in a serial design, such as recurrent events. With this, we use a different frailty term for each recurrent event time within a cluster (person, in infectious diseases) and use a copula function to describe the association between these frailty terms. By extending the copula function to a higher dimension than two, we can also extend this copula-frailty model to settings in which more than two event times are observed for each participant.

We need to take into account the fact that some of the assumptions made in this paper related to the disease dynamics of the different pathogens under study are questionable. First, Goeyvaerts et al. ${ }^{38}$ have demonstrated that the lifelong immunity assumption for parvovirus B19 is untenable based on serological survey data from different European countries. Nevertheless, Abrams and $\mathrm{Hens}^{39}$ showed that the use of frailty models, and by extension our proposed frailty-copula methodology, is not restricted to the case of immunizing infections. Hence, we consider this extension as an attractive avenue for further research. Second, the estimation of the seroprevalence for hepatitis $\mathrm{A}$ is done under the assumption of time equilibrium. Preliminary analyses based on Belgian hepatitis A serial seroprevalence data indicate that this assumption is probably violated. ${ }^{18}$ Classification of individuals into a seronegative or seropositive group inevitably leads to misclassification of subjects whenever the underlying antibody titer distributions, based on which classification is performed, are overlapping. Mixture models provide a compelling framework to avoid specification of thresholds for classifying individuals, and the application of frailty models in such a context would be an interesting topic for further research.

When using a correlated (copula-) frailty model, we need to rely on parametric baseline hazard functions. Although a non-parametric baseline hazard function is of interest in some applications, it is not feasible in our analysis since it will lead to unidentifiability. Still, we used a piecewise Weibull baseline hazard function for HAV infection in the second data application to get a better fit of the models to the data. We consider this piecewise baseline hazard function as a semi-parametric approach which tends to a non-parametric one if the number of breakpoints (in our case, age categories) goes to infinity. Moreover, despite its flexibility, one needs to make a choice about the age groups in which the baseline hazard function is assumed to be of a specific form. To what extent, the use of this semi-parametric approach leads to identifiability problems might be a subject to investigate in future research.

Last but not least, the estimated baseline hazard functions, graphically depicted in Figure 3, and 4, are modelbased. A comparison of these hazard functions with a nonparametric estimator for the hazard function would be interesting as a graphical tool to assess the performance of the model, and possibly the selection of the imposed bivariate frailty distribution. Nonparametric estimation of the hazard function is typically based on smoothing techniques, and nonparametric estimation of the survival function in the presence of current status data is non-trivial. Sun ${ }^{40}$ describes the use of a kernel-based estimator for the hazard function derived from a nonparametric estimator of the survival function in case of current status data. Nevertheless, kernel smoothing implies a (data-driven) selection of a kernel bandwidth to avoid overfitting the data. To our knowledge, not a lot of research has been done on the 
selection of bandwidths in this very specific context. Hence, this endeavor is considered beyond the scope of this manuscript as it would be an interesting future research topic. 
TAB LE 1 Bivariate additive correlated gamma frailty model with Gompertz baseline hazards $\lambda_{i 0}\left(t_{i j}^{*}\right)=a_{i} \exp \left(b_{i} t_{i j}^{*}\right)$ : Empirical mean, empirical standard error (SE), mean squared error (MSE), and median values for the additive gamma frailty, Frank copula-frailty, Gaussian copula-frailty, Clayton copula-frailty, and Gumbel copula-frailty model applied to 300 simulation dataset (sample size $n=3791$ for each data set)

\begin{tabular}{|c|c|c|c|c|c|c|c|}
\hline$\theta, \phi$ & $\begin{array}{l}\text { True } \\
\text { value }\end{array}$ & Report & $\begin{array}{c}\text { Additive } \\
\text { gamma frailty }\end{array}$ & $\begin{array}{c}\text { Frank } \\
\text { copula-frailty }\end{array}$ & $\begin{array}{c}\text { Gaussian } \\
\text { copula-frailty }\end{array}$ & $\begin{array}{c}\text { Clayton } \\
\text { copula-frailty }\end{array}$ & $\begin{array}{c}\text { Gumbel } \\
\text { copula-frailty }\end{array}$ \\
\hline \multirow[t]{4}{*}{$a_{1}$} & 0.0050 & Mean & 0.0050 & 0.0055 & 0.0049 & 0.0055 & 0.0054 \\
\hline & & SE & 0.0006 & 0.0005 & 0.0006 & 0.0005 & 0.0005 \\
\hline & & MSE & 3.7E-7 & $4.9 \mathrm{E}-7$ & $4.4 \mathrm{E}-7$ & $5.0 \mathrm{E}-7$ & $4.9 \mathrm{E}-7$ \\
\hline & & Median & 0.0050 & 0.0055 & 0.0049 & 0.0055 & 0.0054 \\
\hline \multirow[t]{4}{*}{$b_{1}$} & 0.0200 & Mean & 0.0208 & 0.0157 & 0.0250 & 0.0158 & 0.0170 \\
\hline & & SE & 0.0091 & 0.0030 & 0.0129 & 0.0030 & 0.0030 \\
\hline & & MSE & $8.2 \mathrm{E}-5$ & $2.8 \mathrm{E}-5$ & 0.0002 & 2.7E-5 & $1.8 \mathrm{E}-5$ \\
\hline & & Median & 0.0180 & 0.0153 & 0.0210 & 0.0156 & 0.0175 \\
\hline \multirow[t]{4}{*}{$a_{2}$} & 0.0080 & Mean & 0.0080 & 0.0082 & 0.0078 & 0.0082 & 0.0083 \\
\hline & & SE & 0.0009 & 0.0007 & 0.0010 & 0.0008 & 0.0009 \\
\hline & & MSE & $8.8 \mathrm{E}-7$ & $5.9 \mathrm{E}-7$ & $1.1 \mathrm{E}-6$ & $6.8 \mathrm{E}-7$ & $7.9 \mathrm{E}-7$ \\
\hline & & Median & 0.0081 & 0.0082 & 0.0080 & 0.0082 & 0.0083 \\
\hline \multirow[t]{4}{*}{$b_{2}$} & 0.0250 & Mean & 0.0246 & 0.0250 & 0.0304 & 0.0277 & 0.0230 \\
\hline & & SE & 0.0140 & 0.0043 & 0.0186 & 0.0080 & 0.0045 \\
\hline & & MSE & 0.0002 & $1.9 \mathrm{E}-5$ & 0.0004 & 7.1E-5 & $2.4 \mathrm{E}-5$ \\
\hline & & Median & 0.0200 & 0.0249 & 0.0261 & 0.0266 & 0.0234 \\
\hline \multirow[t]{4}{*}{$\alpha_{1}$} & 0.9500 & Mean & 1.2925 & 1.3915 & 1.0978 & 1.3319 & 1.1606 \\
\hline & & SE & 0.5724 & 0.3702 & 1.0489 & 0.3406 & 0.3147 \\
\hline & & MSE & 0.4439 & 0.3315 & 1.1183 & 0.2614 & 0.1424 \\
\hline & & Median & 1.3437 & 1.3234 & 0.9213 & 1.3132 & 1.1087 \\
\hline \multirow[t]{4}{*}{$\alpha_{2}$} & 0.8000 & Mean & 1.1921 & 0.7905 & 0.9756 & 0.7237 & 0.9132 \\
\hline & & SE & 0.5595 & 0.1571 & 0.9254 & 0.2328 & 0.2012 \\
\hline & & MSE & 0.4657 & 0.0247 & 0.8843 & 0.0598 & 0.0529 \\
\hline & & Median & 0.6998 & 0.8000 & 0.7581 & 0.6872 & 0.9123 \\
\hline \multirow[t]{4}{*}{$\tau$} & 0.3888 & Mean & 0.5309 & 0.4374 & 0.3943 & 0.4123 & 0.4458 \\
\hline & & SE & 0.2069 & 0.0469 & 0.1238 & 0.0834 & 0.0797 \\
\hline & & MSE & 0.0629 & 0.0045 & 0.0153 & 0.0075 & 0.0095 \\
\hline & & Median & 0.5039 & 0.4418 & 0.3753 & 0.3989 & 0.4419 \\
\hline
\end{tabular}


TABLE 2 Bivariate Gaussian copula-frailty model with Gompertz baseline hazards $\lambda_{i 0}\left(t_{i j}^{*}\right)=a_{i} \exp \left(b_{i} t_{i j}^{*}\right)$ : Empirical mean, empirical standard error (SE), mean squared error (MSE), and median values for the Gaussian copula-frailty, additive gamma frailty, and Frank copula-frailty model applied to 300 simulation datasets (sample size $n=3791$ for each data set).

\begin{tabular}{|c|c|c|c|c|c|c|c|c|}
\hline \multirow[t]{2}{*}{$(\theta, \phi)$} & \multirow[b]{2}{*}{ True } & \multirow[b]{2}{*}{ Report } & \multicolumn{3}{|c|}{$\rho=-0.1000(\tau=-0.0638)$} & \multicolumn{3}{|c|}{$\rho=-0.9000(\tau=-0.7129)$} \\
\hline & & & Gaussian & Additive & Frank & Gaussian & Additive & Frank \\
\hline & value & & copula-frailty & gamma frailty & copula-frailty & copula-frailty & gamma frailty & copula-frailty \\
\hline \multirow[t]{4}{*}{$a_{1}$} & 0.0050 & Mean & 0.0048 & 0.0048 & 0.0054 & 0.0087 & 0.0048 & 0.0053 \\
\hline & & SE & 0.0006 & 0.0007 & 0.0005 & 0.0050 & 0.0007 & 0.0005 \\
\hline & & MSE & $4.2 \mathrm{E}-7$ & $4.6 \mathrm{E}-7$ & $3.8 \mathrm{E}-7$ & $3.8 \mathrm{E}-5$ & $5.0 \mathrm{E}-7$ & $3.3 \mathrm{E}-7$ \\
\hline & & Median & 0.0049 & 0.0049 & 0.0054 & 0.0082 & 0.0049 & 0.0053 \\
\hline \multirow[t]{4}{*}{$b_{1}$} & 0.0200 & Mean & 0.0245 & 0.0250 & 0.0163 & 0.0221 & 0.0269 & 0.0194 \\
\hline & & SE & 0.0144 & 0.0183 & 0.0031 & 0.0047 & 0.0443 & 0.0027 \\
\hline & & MSE & 0.0002 & 0.0004 & $2.3 \mathrm{E}-5$ & $2.7 \mathrm{E}-5$ & 0.0020 & $7.7 \mathrm{E}-6$ \\
\hline & & Median & 0.0206 & 0.0194 & 0.0162 & 0.0214 & 0.0177 & 0.0194 \\
\hline \multirow[t]{4}{*}{$a_{2}$} & 0.0100 & Mean & 0.0110 & 0.0115 & 0.0101 & 0.1474 & 9.6565 & 0.0101 \\
\hline & & SE & 0.0041 & 0.0045 & 0.0014 & 0.4009 & 166.8253 & 0.0014 \\
\hline & & MSE & $1.8 \mathrm{E}-5$ & $2.2 \mathrm{E}-5$ & $2.0 \mathrm{E}-6$ & 0.1791 & 27830.9800 & $2.0 \mathrm{E}-6$ \\
\hline & & Median & 0.0100 & 0.0104 & 0.0100 & 0.1163 & 0.0101 & 0.0100 \\
\hline \multirow[t]{4}{*}{$b_{2}$} & 0.0025 & Mean & 0.0063 & 0.0091 & -0.0003 & 0.0003 & 0.0140 & 0.0022 \\
\hline & & SE & 0.0218 & 0.0253 & 0.0071 & 0.0114 & 0.0779 & 0.0068 \\
\hline & & MSE & 0.0005 & 0.0007 & $5.9 \mathrm{E}-5$ & 0.0001 & 0.0062 & $4.7 \mathrm{E}-5$ \\
\hline & & Median & 0.0010 & 0.0031 & 0.0009 & -0.0014 & 0.0032 & 0.0021 \\
\hline \multirow[t]{4}{*}{$\alpha_{1}$} & 0.8000 & Mean & 1.2421 & $3.4 \mathrm{E}+7$ & 1.0676 & 0.7183 & $3.2 \mathrm{E}+7$ & 0.7555 \\
\hline & & SE & 1.9659 & $4.2 \mathrm{E}+8$ & 0.2484 & 0.1561 & $3.3 \mathrm{E}+8$ & 0.1187 \\
\hline & & MSE & 4.0473 & $1.7 \mathrm{E}+17$ & 0.1331 & 0.0309 & $1.1 \mathrm{E}+17$ & 0.0160 \\
\hline & & Median & 0.8110 & 0.9300 & 1.0529 & 0.7497 & 1.0495 & 0.7465 \\
\hline \multirow[t]{4}{*}{$\alpha_{2}$} & 0.2000 & Mean & 0.5104 & $2.5 \mathrm{E}+7$ & 0.2324 & 0.2573 & $2.2 \mathrm{E}+7$ & 0.2120 \\
\hline & & SE & 1.4774 & $4.3 \mathrm{E}+8$ & 0.0633 & 0.0824 & $3.8 \mathrm{E}+8$ & 0.0518 \\
\hline & & MSE & 2.2717 & $1.8 \mathrm{E}+17$ & 0.005 & 0.0101 & $1.4 \mathrm{E}+17$ & 0.0028 \\
\hline & & Median & 0.2078 & 0.1819 & 0.2185 & 0.2677 & 0.1961 & 0.2009 \\
\hline \multirow[t]{4}{*}{$\rho$} & - & Mean & -0.1296 & 0.0015 & - & -0.8981 & 0.0003 & - \\
\hline & & SE & 0.1320 & 0.0049 & - & 0.0762 & 0.0006 & - \\
\hline & & MSE & 0.0182 & 0.0103 & - & 0.0058 & $8.1 \mathrm{E}-1$ & - \\
\hline & & Median & -0.1065 & 0.0001 & - & -0.9121 & $4.1 \mathrm{E}-5$ & - \\
\hline \multirow[t]{4}{*}{$\tau$} & - & Mean & -0.0868 & 0.0005 & -0.0630 & -0.7291 & 0.0002 & -0.6628 \\
\hline & & SE & 0.1105 & 0.0024 & 0.0608 & 0.1088 & 0.0011 & 0.0323 \\
\hline & & MSE & 0.0127 & 0.0041 & 0.0037 & 0.0121 & 0.5085 & 0.0036 \\
\hline & & Median & -0.0680 & 0.0002 & -0.0640 & -0.7310 & 0.0002 & -0.6555 \\
\hline
\end{tabular}


TAB LE 3 Bivariate Frank copula-frailty model with Gompertz baseline hazards $\lambda_{i 0}\left(t_{i j}^{*}\right)=a_{i} \exp \left(b_{i} t_{i j}^{*}\right)$ : Empirical mean, empirical standard error (SE), mean squared error (MSE), and median values for the Frank copula-frailty, additive gamma frailty, and Gaussian copula-frailty model applied to 300 simulation datasets (sample size $n=3791$ for each data set).

\begin{tabular}{|c|c|c|c|c|c|c|c|c|}
\hline \multirow[t]{2}{*}{$(\theta, \phi)$} & \multirow[b]{2}{*}{ True } & \multirow[b]{2}{*}{ Report } & \multicolumn{3}{|c|}{$\tau=-0.2240$} & \multicolumn{3}{|c|}{$\tau=-0.6430$} \\
\hline & & & Frank & Additive & Gaussian & Frank & Additive & Gaussian \\
\hline & value & & copula-frailty & gamma frailty & copula-frailty & copula-frailty & gamma-frailty & copula-frailty \\
\hline \multirow[t]{4}{*}{$a_{1}$} & 0.0050 & Mean & 0.0053 & 0.0048 & 0.0050 & 0.0053 & 0.0048 & 0.0049 \\
\hline & & SE & 0.0005 & 0.0008 & 0.0006 & 0.0005 & 0.0009 & 0.0006 \\
\hline & & MSE & $3.6 \mathrm{E}-7$ & $6.9 \mathrm{E}-7$ & $3.5 \mathrm{E}-7$ & $3.5 \mathrm{E}-7$ & $7.6 \mathrm{E}-7$ & $3.2 \mathrm{E}-7$ \\
\hline & & Median & 0.0053 & 0.0050 & 0.0050 & 0.0053 & 0.0049 & 0.0049 \\
\hline \multirow[t]{4}{*}{$b_{1}$} & 0.0200 & Mean & 0.0181 & 0.0300 & 0.0231 & 0.0187 & 0.0390 & 0.0236 \\
\hline & & SE & 0.0025 & 0.0418 & 0.0138 & 0.0027 & 0.1797 & 0.0089 \\
\hline & & MSE & $9.8 \mathrm{E}-6$ & 0.0018 & 0.0002 & $9.2 \mathrm{E}-6$ & 0.0326 & $9.3 \mathrm{E}-5$ \\
\hline & & Median & 0.0181 & 0.0167 & 0.0197 & 0.0192 & 0.0157 & 0.0214 \\
\hline \multirow[t]{4}{*}{$a_{2}$} & 0.0100 & Mean & 0.0101 & 0.0156 & 0.0109 & 0.0103 & 0.0134 & 0.0107 \\
\hline & & SE & 0.0013 & 0.0728 & 0.0062 & 0.0015 & 0.0184 & 0.0027 \\
\hline & & MSE & $1.7 \mathrm{E}-6$ & 0.0053 & $3.9 \mathrm{E}-5$ & $2.2 \mathrm{E}-6$ & 0.0003 & $7.7 \mathrm{E}-6$ \\
\hline & & Median & 0.0100 & 0.0099 & 0.0098 & 0.0101 & 0.0102 & 0.0102 \\
\hline \multirow[t]{4}{*}{$b_{2}$} & 0.0025 & Mean & 0.0030 & 0.0104 & 0.0071 & 0.0036 & 0.0139 & 0.0073 \\
\hline & & SE & 0.0056 & 0.0416 & 0.0252 & 0.0064 & 0.0436 & 0.0172 \\
\hline & & MSE & 3.1E-05 & 0.0018 & 0.0007 & $4.2 \mathrm{E}-5$ & 0.0020 & 0.0003 \\
\hline & & Median & 0.0026 & 0.0027 & 0.0018 & 0.0026 & 0.0010 & 0.0031 \\
\hline \multirow[t]{4}{*}{$\alpha_{1}$} & 0.8000 & Mean & 0.8642 & 18.9557 & 1.1979 & 0.8207 & 8.2026 & 0.7043 \\
\hline & & SE & 0.1081 & 90.0369 & 1.4383 & 0.1036 & 22.8329 & 0.2919 \\
\hline & & MSE & 0.0158 & 8409.254 & 2.2199 & 0.0111 & 574.4028 & 0.0884 \\
\hline & & Median & 0.8461 & 1.2535 & 0.8249 & 0.8000 & 1.4556 & 0.7548 \\
\hline \multirow[t]{4}{*}{$\alpha_{2}$} & 0.2000 & Mean & 0.1980 & 3.0945 & 0.3379 & 0.1934 & 1.6977 & 0.2235 \\
\hline & & SE & 0.0298 & 20.475 & 0.4465 & 0.0308 & 7.5430 & 0.1293 \\
\hline & & MSE & 0.0009 & 426.2056 & 0.2177 & 0.0010 & 58.9507 & 0.0172 \\
\hline & & Median & 0.2000 & 0.2009 & 0.2167 & 0.2000 & 0.1971 & 0.2029 \\
\hline \multirow[t]{4}{*}{$\tau$} & - & Mean & -0.2273 & $5.80 \mathrm{E}-05$ & -0.2799 & -0.6459 & 0.0001 & -0.6682 \\
\hline & & SE & 0.0292 & 0.0011 & 0.1495 & 0.0125 & 0.0010 & 0.1448 \\
\hline & & MSE & 0.0009 & 0.0502 & 0.0254 & 0.0002 & 0.4136 & 0.0215 \\
\hline & & Median & -0.2242 & $8.60 \mathrm{E}-05$ & -0.2381 & -0.6432 & 0.0001 & -0.6581 \\
\hline
\end{tabular}


TAB LE 4 Parameter and standard error estimates for the VZV-PVB19 analysis using Weibull baseline hazard functions $\left(\lambda_{i 0}\left(t_{i}\right)=\rho_{i} \zeta_{i} t_{i}^{\rho_{i}-1}\right), i=1,2$ and considering the Gaussian copula-frailty model, the Frank copula-frailty model, the additive correlated frailty model, the Clayton copula-frailty model, and the Gumbel copula-frailty model with marginal gamma frailty distributions. Analysis done using information on 2975 individuals.

\begin{tabular}{|c|c|c|c|c|c|}
\hline & $\begin{array}{c}\text { Gaussian } \\
\text { copula-frailty }\end{array}$ & $\begin{array}{c}\text { Frank } \\
\text { copula-frailty }\end{array}$ & $\begin{array}{c}\text { Additive } \\
\text { gamma frailty }\end{array}$ & $\begin{array}{c}\text { Clayton } \\
\text { copula-frailty }\end{array}$ & $\begin{array}{c}\text { Gumbel } \\
\text { copula-frailty }\end{array}$ \\
\hline$\zeta_{1}$ & $0.3302(0.0666)$ & $0.3281(0.0660)$ & $0.4821(0.0620)$ & $0.3321(0.0667)$ & $0.3256(0.0661)$ \\
\hline$\rho_{1}$ & $1.4180(0.2692)$ & $1.4392(0.2698)$ & $0.9175(0.1034)$ & $1.3911(0.4176)$ & $1.4445(0.2727)$ \\
\hline$\zeta_{2}$ & 0.0415 (0.0173) & $0.0423(0.0175)$ & $0.0456(0.0179)$ & $0.0421(0.0175)$ & $0.0407(0.0171)$ \\
\hline$\rho_{2}$ & $2.2910(0.4218)$ & $2.2738(0.4183)$ & $2.1635(0.3733)$ & $2.2739(0.4176)$ & $2.3177(0.4291)$ \\
\hline$\alpha_{1}$ & $1.2320(0.3948)$ & $1.2007(0.3749)$ & $2.7399(0.8177)$ & $1.2800(0.4181)$ & $1.1961(0.3751)$ \\
\hline$\alpha_{2}$ & $0.2466(0.0552)$ & $0.2487(0.0557)$ & $0.2654(0.0559)$ & $0.2490(0.0556)$ & $0.2430(0.0546)$ \\
\hline$\sigma_{1}^{2}$ & $0.8117(0.2601)$ & $0.8329(0.2601)$ & $0.3650(0.1089)$ & $0.7813(0.2552)$ & $0.8361(0.2622)$ \\
\hline$\sigma_{2}^{2}$ & 4.0544 (0.9073) & $4.0207(0.9010)$ & $3.7685(0.7936)$ & $4.0207(0.8965)$ & $4.1149(0.9250)$ \\
\hline$\rho$ & $0.2306(0.0700)$ & - & $0.3103(0.0495)$ & - & - \\
\hline$\tau$ & $0.1481(0.0458)$ & $0.1841(0.0530)$ & $0.1492(0.0121)$ & $0.0727(0.0303)$ & $0.2000(0.0535)$ \\
\hline$-2 L L$ & 4480.0290 & 4478.7110 & 4489.0850 & 4483.9710 & 4477.5580 \\
\hline$A I C$ & 4494.0290 & 4492.7110 & 4503.0850 & 4497.9710 & 4491.5580 \\
\hline
\end{tabular}


TAB LE 5 Parameter and standard error estimates for the VZV-HAV analysis using Weibull baseline hazard function for VZV and piecewise Weibull baseline hazard with cut-off point at the age of 30 for HAV, considering the Gaussian copula-frailty model, the Frank copula-frailty model, the additive correlated frailty model, the Clayton copula-frailty model, and the Gumbel copula-frailty model with marginal gamma frailty distributions. The analysis was done using information on 3256 individuals.

\begin{tabular}{|c|c|c|c|c|c|}
\hline & $\begin{array}{c}\text { Gaussian } \\
\text { copula-frailty }\end{array}$ & $\begin{array}{c}\text { Frank } \\
\text { copula-frailty }\end{array}$ & $\begin{array}{c}\text { Additive } \\
\text { gamma frailty }\end{array}$ & $\begin{array}{c}\text { Clayton } \\
\text { copula-frailty }\end{array}$ & $\begin{array}{c}\text { Gumbel } \\
\text { copula-frailty }\end{array}$ \\
\hline$\zeta_{1}$ & $0.3430(0.0568)$ & $0.3415(0.0567)$ & $0.5303(0.0468)$ & $0.3444(0.0569)$ & $0.3418(0.0568)$ \\
\hline$\rho_{1}$ & $1.2927(0.2214)$ & $1.3022(0.2225)$ & $0.6582(0.0590)$ & $1.2839(0.2199)$ & $1.2986(0.2226)$ \\
\hline$\zeta_{21}$ & $0.0753(0.0143)$ & $0.0752(0.0142)$ & $0.0752(0.0143)$ & $0.0752(0.0142)$ & $0.0752(0.0143)$ \\
\hline$\varrho_{21}$ & $0.3558(0.0721)$ & $0.3526(0.0703)$ & $0.3547(0.0712)$ & $0.3535(0.0709)$ & $0.3588(0.0724)$ \\
\hline$\zeta_{22}$ & $0.0069(0.0052)$ & $0.0078(0.0049)$ & $0.0075(0.0051)$ & $0.0076(0.0050)$ & $0.0069(0.0052)$ \\
\hline$\varrho_{22}$ & $1.5733(0.3005)$ & $1.5233(0.2110)$ & $1.5524(0.2573)$ & $1.5397(0.2471)$ & $1.6037(0.3151)$ \\
\hline$\alpha_{1}$ & $1.4412(0.4644)$ & $1.4214(0.4568)$ & $25.3384(41.4732)$ & $1.4602(0.4777)$ & $1.4301(0.4628)$ \\
\hline$\alpha_{2}$ & 6.6614 (14.7339) & $23.6990(106.8622)$ & 9.8890 (32.9951) & $14.1300(62.4160)$ & $4.8712(11.4205)$ \\
\hline$\sigma_{1}^{2}$ & $0.6939(0.2253)$ & $0.7036(0.2261)$ & $0.0395(0.0646)$ & $0.6848(0.2240)$ & $0.6993(0.2263)$ \\
\hline$\sigma_{2}^{2}$ & $0.1501(0.4541)$ & $0.0422(0.1903)$ & $0.1011(0.3374)$ & $0.0408(0.3126)$ & $0.2053(0.4813)$ \\
\hline$\rho$ & $0.2079(0.4335)$ & - & $0.6246(1.3499)$ & - & - \\
\hline$\tau$ & $0.1333(0.2821)$ & 0.3709 (0.8987) & $0.4283(0.0090)$ & $0.0654(0.2565)$ & $0.1425(0.2283)$ \\
\hline$-2 L L$ & 4546.5150 & 4546.1770 & 4555.7810 & 4546.7490 & 4546.5340 \\
\hline$A I C$ & 4564.5150 & 4564.1770 & 4573.7810 & 4564.7490 & 4564.5340 \\
\hline
\end{tabular}




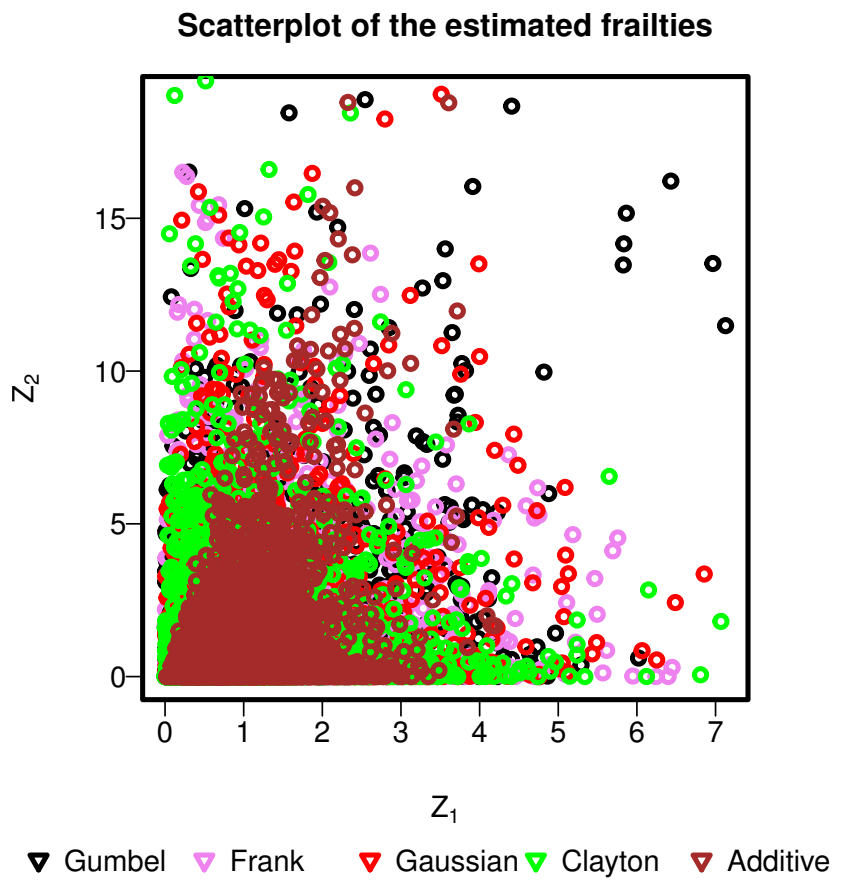

FIGURE 1 Scatter plot of estimated frailties $\left(Z_{1}, Z_{2}\right)$ under different models: The Gumbel copula-frailty model with the could of points in black, the Frank copula-frailty model with violet points, the Gaussian copula-frailty model with red cloud, the Clayton copula-frailty model with green points, and the additive correlated gamma frailty model with brown cloud. 


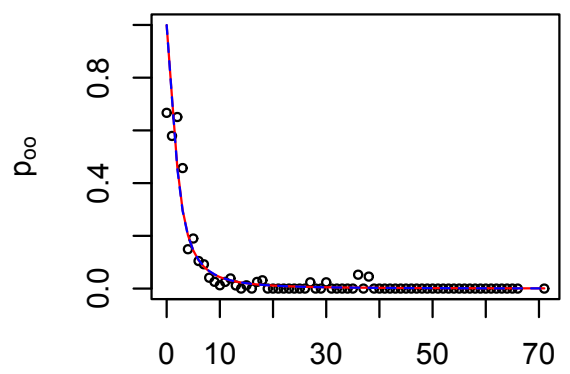

age

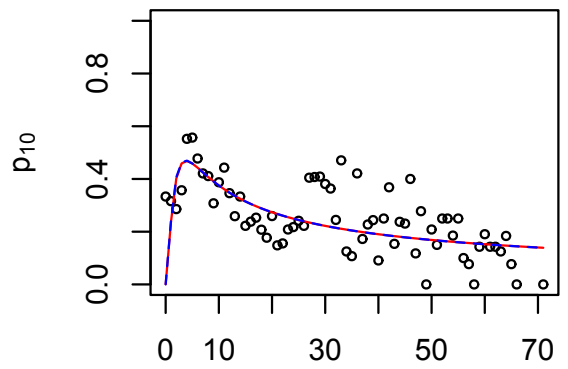

age

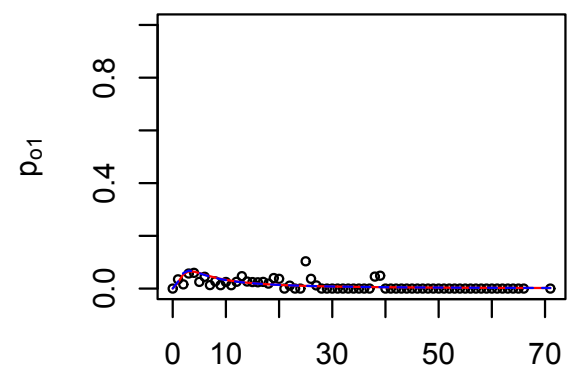

age

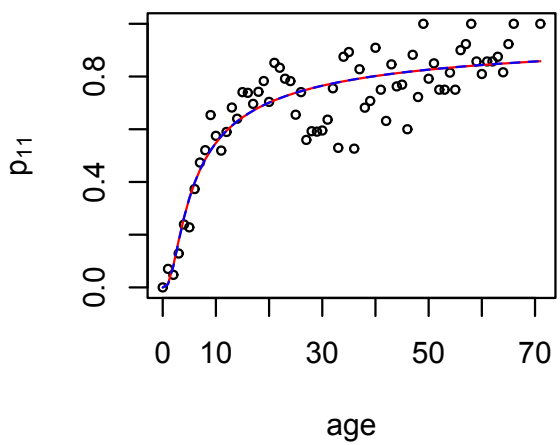

FIGURE 2 Plot of the joint probabilities of VZV and PVB19 infections with the Gumbel copula-frailty model fit (Weibull baseline hazards) in red, and the Frank copula-frailty model fit in dotted green. $p_{00}$ refers to the joint probability of no past infection for either disease, that is the age-dependent proportion of people who are still susceptible to both infections (left upper panel); $p_{10}$ refers to past and no past infection for VZV and PVB19, respectively, that is the age-dependent proportion of people who are already infected by VZV and still susceptible to PVB19 (left lower panel); $p_{01}$ refers to no past and past infection for VZV and PVB19, respectively, that is the age-dependent proportion of people who are still susceptible to VZV and already infected by PVB19 (right upper panel); and $p_{11}$ refers to past infection for both diseases, that is the age-dependent proportion of people who are already infected by both infections (right lower panel). 


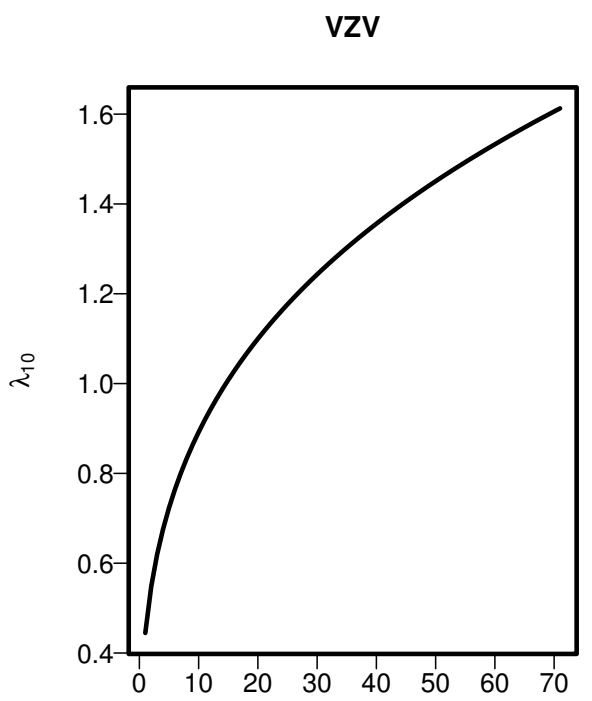

age

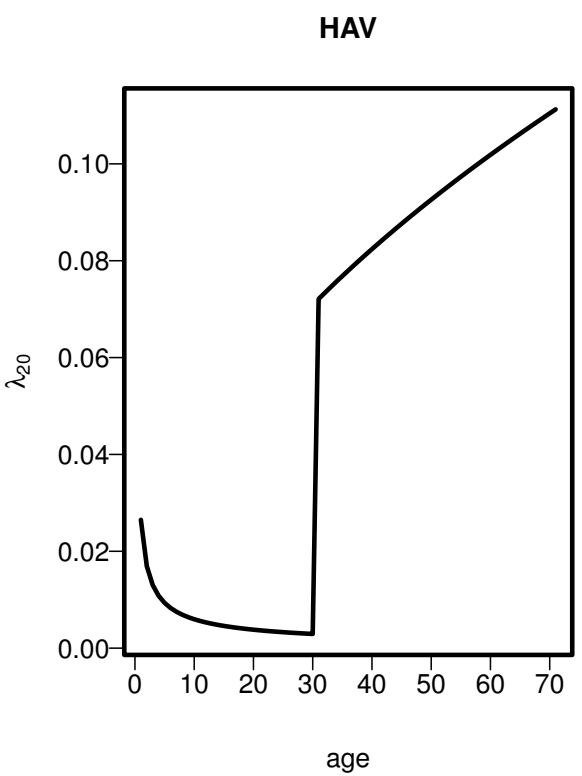

FIGURE 3 Plot of the baseline hazard functions for the two diseases: VZV (in the left panel) and HAV (in the right panel) using estimated values from the "best" model (the Frank copula-frailty model) in terms of AIC. 


\section{Acknowledgements}

We acknowledge Prof. Dr. Heidi Theeten for providing us with the serological data set.

\section{Conflict of interest}

The authors have declared no conflict of interest.

\section{Supporting information}

Additional supporting information may be found online in the Supporting Information section at the end of this article. The data that support the findings of this study are openly available in [repository name e.g "figshare"] at http://doi.org/[doi], reference number [reference number].

\section{References}

1. Klein JP, Moeschberger ML. Survival analysis: techniques for censored and truncated data. Springer Science \& Business Media; 2006.

2. Wang W, Ding AA. On assessing the association for bivariate current status data. Biometrika. 2008;87(4):879-893.

3. Kalbfeisch JD, Prentice RL. The statistical analysis of failure time data. John Wiley \& Sons; 2011.

4. Shil JH, Louis TA. Inferences on the association parameter in copula models for bivariate survival data. Biometrics. 1995;51(4):1384-1399. doi:10.2307/2533269.

5. Romeo JS, Meyer R, Gallardo DI. Bayesian bivariate survival analysis using the power variance function copula. Lifetime Data Anal. 2018;24(2):355-383. doi: 10.1007/s10985-017-9396-1.

6. Andersen EW. Two-stage estimation in copula models used in family studies. Lifetime Data Anal. 2005;11(3):33350. doi:10.1007/s10985-005-2966-7.

7. Prenen L, Braekers R, Duchateau L. Extending the Archimedean copula methodology to model multivariate survival data grouped in clusters of variable size. J R Stat Soc Series B Stat Methodol. 2017;79(2),483-505.

8. Lawless JF, Yilmaz YE. Semiparametric estimation in copula models for bivariate sequential survival times. Biom J. 2011;53(5):779-96. doi:10.1002/bimj.201000131.

9. Meyer R, Romeo JS. Bayesian semiparametric analysis of recurrent failure time data using copulas. Biom J. 2015;57(6):9821001. doi: 10.1002/bimj.201400125.

10. Goethals K, Janssen P, Duchateau L. Frailty models and copulas: similarities and differences. J Appl Stat. 2008;35(9):10711079.

11. Wienke A. Frailty models in survival analysis. Chapman and Hall/CRC; 2010.

12. Duchateau L, Janssen P. The frailty model. Springer Science \& Business Media; 2007.

13. Balakrishnan N, Peng Y. Generalized gamma frailty model. Stat Med. 2006;25(16):2797-816. doi:10.1002/sim.2375.

14. Hougaard P. Analysis of multivariate survival data. Springer Science \& Business Media; 2012.

15. Hens N, Wienke A, Aerts M, Molenberghs $G$. The correlated and shared gamma frailty model for bivariate current status data: an illustration for cross-sectional serological data. Stat Med. 2009;28(22):2785-800. doi: 10.1002/sim.3660.

16. Yashin Al, Vaupel JW, lachine IA. Correlated individual frailty: an advantageous approach to survival analysis of bivariate data. Math Popul Stud. 1995;5(2):145-59, 183. doi:10.1080/08898489509525394.

17. Glidden DV. A two-stage estimator of the dependence parameter for the Clayton-Oakes model. Lifetime Data 
Anal. 2000;6(2):141-56.

18. Hens N, Shkedy Z, Aerts M, Faes C, VanDamme P, Beutels P. Modeling infectious disease parameters based on serological and social contact data: a modern statistical perspective. Springer Science \& Business Media; 2012.

19. Debnath J, Dahiya R. Theorems on multidimensional Laplace transform for solution of boundary value problems. Comput Math Appl. 1989;18(12):1033-1056.

20. Sun J. The statistical analysis of interval-censored failure time data. Springer Science \& Business Media; 2007.

21. Iachine I. Identifiability of bivariate frailty models. Preprint 2004;5. http://citeseerx . ist.psu . edu/viewdoc/ download?doi=10.1 1.138.2944\&rep=rep1\&type=pdf. Accessed May 10, 2019.

22. Nelsen RB. An introduction to copulas. Spriner Science \& Business Media; 2007.

23. Bárdossy A, Li J. Geostatistical interpolation using copulas. Water Resour Res. 2008;44(7):1-15.

24. Danaher PJ, Smith MS. Modeling multivariate distributions using copulas: applications in marketing. Market Sci. 2011;30(1):4-21.

25. de Leon AR, Wu B. Copula-based regression models for a bivariate mixed discrete and continuous outcome. Stat Med. 2011;30(2):175-85. doi: 10.1002/sim.4087.

26. Song PXK, Li M, Yuan Y. Joint regression analysis of correlated data using Gaussian copulas. Biometrics. 2009;65(1):6068.

27. Frahm G, Junker M, Schmidt R. Estimating the tail-dependence coefficient: properties and pitfalls. Insur Math Econ. 2005;37(1):80-100.

28. Shi P, Yang L. Pair copula constructions for insurance experience rating. J Am Stat Assoc. 2018;113(521):122-133.

29. Meyer C. The bivariate normal copula. Commun Stat Theory Methods. 2013;42(13):2402-2422.

30. Schepsmeier U, Stöber J. Derivatives and Fisher information of bivariate copulas. Stat Pap. 2014;55(2):525-542.

31. Mahfoud M, Michael M. Bivariate Archimedean copulas: an application to two stock market indices. BMI Paper. 2012. https://beta.vu.nl/nl/Images/werkstuk-mahfoud_tcm235-277460.pdf. Accessed Apr 10, 2019.

32. Abrams S, Aerts M, Molenberghs G, Hens N. Parametric overdispersed frailty models for current status data. Biometrics. 2017;73(4):1388-1400.

33. Akaike H. Information theory and an extension of the maximum likelihood principle. Breakthroughs in statistics Springer. 1992; 610-624.

34. Munda M, Rotolo F, Legrand C. Parfm: parametric frailty models in R. J Stat Softw. 2012;51(11):1-20.

35. Grothe O, Nicklas S. Vine constructions of Lévy copulas. J Multivar Anal. 2013;119:1-15.

36. Aas K, Czado C, Frigessi A, Bakken H. Pair-copula constructions of multiple dependence. Insur Math Econ. 2009;44(2):182198.

37. Aas K, Berg D. Models for construction of multivariate dependence-a comparison study. Copulae and Multivariate Probability Distributions in Finance. 2009;15(7-8):639-659. doi:10.1080/13518470802588767.

38. Goeyvaerts N, Hens N, Aerts M, Beutels P. Model structure analysis to estimate basic immunological processes and maternal risk for parvovirus B19. Biostatistics. 2010;12(2):283-302.

39. Abrams S, Hens N. Modeling individual heterogeneity in the acquisition of recurrent infections: an application to parvovirus B19. Biostatistics. 2014;16(1):129-142.

40. Sun J. The Statistical Analysis of Interval-Censored Failure Time Data. Springer: New York; 2006. 